\title{
Évaluation agronomique des variétés de haricot volubile riches en micronutriments dans un système intégré d'Agroforesterie sur deux sols contrastés à l'Est de la RD Congo
}

Ntamwira Bagula Jules ${ }^{1,2 *}$, Mirindi Cirhuza Télesphore ${ }^{1}$, Pyame Mwarabu Lolonga Dieudonne², Dhed'a Djailo Benoit ${ }^{2}$, Bumba Mariam Espérance ${ }^{3}$, Moango Manga Adrien², Kazadi Wakenge Josué ${ }^{4}$ et Kanyenga Lubobo Antoine $e^{4,5}$

${ }^{1}$ Institut National pour l'Étude et la Recherche Agronomiques, BP 2037 Kinshasa 1, Station de Mulungu, RD Congo. telecirhu@yahoo.fr

2Facultés des Sciences et de Gestion de Ressources Renouvelables, Université de Kisangani, BP 2012, Kisangani, RD Congo. pyamed@yahoo.fr , dhedadjailo@gmail.com ,moangoadrien@gmail.com

3Bioversity, Bukavu, Sud, DR Congo. Bumba.marie@gmail.com

${ }^{4}$ CIAT-HarvestPlus, Bureau de Bukavu, RD Congo. kazadijosue@gmail.com; a.k.lubobo@cgiar.org

${ }^{5}$ Faculté des Sciences Agronomiques, Université de Lubumbashi, BP 1825, Lubumbashi, RD Congo.

kanyengalub@gmail.com

*Auteur correspondent email : ingjules2007@yahoo.fr , Tel : +243993703098

Original submitted in on $5^{\text {th }}$ April 2017. Published online at www.m.elewa.org on $30^{\text {th }}$ June 2017 https://dx.doi.org/10.4314/jab.v114i1.10

\section{RESUME}

Objectif : L'objectif de cette étude était d'évaluer différentes variétés de haricots riches en fer et zinc dans un système intégré de type «jachère-herbage agro-forestière».

Méthodologie et résultats : Le dispositif expérimental était le split plot avec comme parcelles principales le type de jachère-herbage agro-forestière, elles étaient subdivisées en quatre sous parcelles secondaires chacune correspondant à une variété de haricot. Les parcelles étaient au total onze et constituaient les différents traitements : T00 (témoin : 0 herbage-arbres, 0 NPK et 0 Fumier) ; T0 (NPK+Fumier), T1 à T9 issus de la combinaison herbes $x$ arbres+NPK et fumier. Les 11 parcelles principales ont constitué un bloc répété cinq fois par site. Le fumier (20 t MS/ha) et les micros doses d'engrais NPK (50 kg.ha-1) étaient appliqués. Des différences ont été observées entre les traitements et les deux sites. Le traitement NPK+Fumier (TO) a donné les rendements en graines de haricots et les teneurs en zinc et en fer dans les graines de haricots les plus élevés dans les deux sites, dans le site avec sol fertile le rendement était de 1144,2 kg.ha-1 de graines et dans le site avec sol pauvre de 260, 6kg.ha-1 de graines). Les traitements T00 et NPK + Fumier+ jachère-herbage agro-forestière ont donné des rendements les plus bas (12,2 et 4,7 kg.ha-1) respectivement dans les sols pauvre et fertile. Les scores de maladies étaient bas, les variétés ont affiché une réaction intermédiaire.

Conclusion et application de résultats : L'application de la microdose d'engrais et la jachère-herbage agroforestière ont augmenté le rendement en graines et la teneur en micronutriments dans les graines de haricots. En plus, cet effet était plus prononcé sur sol pauvre que sur sol fertile. L'application de microdoses d'engrais minéral et organique est l'un d'importantes pratiques pour améliorer le rendement de haricots dans le sol pauvre de Mushinga.

Mots clés : Arbustes, fertilité du sol, graminées, légumineuses, haricots biofortifiés, rendement. 
Agronomic evaluation of different climbing beans varieties rich in micronutrient in an integrated Agroforestry systems in two contrasting soils in south Kivu, Eastern DR Congo

\section{ABSTRACT}

Objective: The objective of this study was to evaluate different varieties of climbing beans (Phaseolus vulgaris L.) rich in iron and zinc in an integrated agroforestry system.

Methodology and Results: The experiments were established in two sites using a split-plot design, with the main plot treatments consisting of agroforestry-fallow types. These plots were 11 in total: T00 (control: 0 grasses, trees, NPK and manure); T0 (NPK+manure), T1 to T9 from the combination grass-trees+NPK and manure and were repeated five times per site. They were subdivided into 4 subplots, each corresponding to one variety of beans. Manure (20 t DM/ha) and micro doses of NPK fertilizer (50 kg ha-1) were applied. Differences were observed between treatment and between sites. The treatment with NPK+manure (T0) gave the highest grains yields and content in zinc and iron in the grain beans in the two sites, $1144.2 \mathrm{~kg}$ ha- 1 for site with fertile soil and $260.6 \mathrm{~kg}$ ha-1 in poor soil. The T00 treatments and NPK+manure+agroforestry fallow grassland gave the lowest bean grain yields (12.2 and $4.7 \mathrm{~kg}$ ha-1) respectively in the poor and fertile soils. Disease scores were low, varieties showed intermediate reaction.

Conclusion and application of results: Bean grain yield and micronutrient content in the grain beans were enhanced with application of microdose of fertilizers and agroforestry-fallow grassland. In addition, this effect was more pronounced on poor soil than on fertile soil. The application of microdose of mineral and organic fertilizer is one of the important practices for improving bean yield in the poor soil of Mushinga.

Keywords: Biofortified beans, grasses, legumes, shrubs, soil fertility, yield.

\section{INTRODUCTION}

Dans le Kivu montagneux à forte population, les agriculteurs recourent à l'association de cultures telle que les haricots-bananiers, haricots-maïs-maniocs, bananiers-taros, etc. du fait de l'insuffisance de terres cultivables (CIALCA, 2010 ; Ntamwira et al., 2014). La superficie moyenne de l'exploitation agropastorale par ménage étant de moins de 0,5 ha (CIALCA, 2010). Cependant, dans les agrosystèmes à base du haricot, les rendements en graines de haricots (400 à $800 \mathrm{Kg} \mathrm{ha}^{-1}$ ) restent encore faibles (Bouwmeester et al., 2009). Pourtant les résultats de recherche ont montré que plus de 2.000 à $3.000 \mathrm{~kg}$ ha- 1 de haricots nains et de 4.000 à $6.000 \mathrm{~kg}$ ha- 1 de haricots volubiles peuvent être produits (Kanyenga et al., 2016). Les causes de ces faibles rendements dans la province du Sud Kivu sont nombreuses, il s'agit principalement de pratiques agricoles inappropriées avec comme conséquences l'épuisement du sol, les maladies et le changement climatique (Bouwmeester et al., 2009; Lunze et al, 2012; Kanyenga et al., 2016 ). La plupart des agriculteurs brûlent les herbes lors de la préparation de terrain pour le semis, ceci peut avoir comme conséquence, la perte de $40 \mathrm{~kg}$ d'N et de $10 \mathrm{~kg}$ de $S$ par hectare à chaque saison culturale (Sanginga \&
Woomer, 2009 ; Civava et al., 2012). Cette situation est aggravée, d'une part, par le fait que les terres cultivées, plus particulièrement celles en pentes, sont de plus en plus touchées par l'érosion due à un ruissèlement massif, celle-ci étant la majeure source de dégradation et de perte de terres arables dans le Kivu montagneux (MASI, 1982). D'autre part, la pratique du labour traditionnel répétée, sur ces terrains en pente plus ou moins forte, et la faible utilisation des intrants agricoles favorisent le phénomène d'érosion et la diminution de la production (Razafindramanan et al., 2012). En plus, cette dégradation continue de sols est corrélée à l'inexistence, en agriculture paysanne africaine, de paquets technologiques d'éco-agriculture efficaces (Pyame, 2015). Si nous voulons améliorer les rendements et utiliser les sols pour produire des cultures de manière durable, d'une part, il nous faut trouver une méthode permettant de maintenir les éléments nutritifs dans le sol. Par exemple, l'association manioc + Brachiaria sp, bien gérée, a bénéficié très fortement au manioc et a permis de doubler ou tripler les rendements par rapport à la culture pure du manioc au Madagascar (Husson et al., 2008). D'autre part, nous devons fournir aux 
cultures les éléments dont le sol n'est pas suffisamment pourvu pour obtenir de bons rendements. Les engrais permettent souvent de doubler et même de tripler les rendements (Sanginga \& Woomer, 2009). Par exemple, les résultats des observations menés par Pypers et al., (2010) dans deux sites à caractéristiques différentes dans la province du Sud-Kivu ont montré que l'ajout d'engrais a augmenté les rendements en grains de haricot de 11 et 24\%. Également, l'application d'engrais et la modification des écartements des cultures ont augmenté les rendements de haricots de $23 \%$ et $21 \%$ par rapport aux pratiques traditionnelles. L'application d'engrais a augmenté les rendements de manioc de près de $20 \%$ (Pypers et al., 2010). Cependant, les travaux récents ont montré que la productivité des sols sous les tropiques baisse même avec l'utilisation continue des engrais chimiques seuls (Kaho et al., 2011). Mulangwa (2001) a montré que rien qu'avec les biomasses de Tithonia diversifolia, des accroissements spectaculaires de rendement du haricot (de l'ordre de $150 \%$ du témoin sans fumure) peuvent être obtenus. Les engrais organiques seuls sont insuffisants actuellement pour compenser le faible niveau des éléments nutritifs dans les sols tropicaux (Kaho et al., 2011). C'est ainsi qu'il a était démontré que la combinaison des biomasses avec les engrais minéraux permettent au haricot de mieux exprimer son potentiel de production. Aussi, la combinaison des engrais minéraux avec du fumier ou du compost améliore l'efficacité des engrais (Sanginga \& Woomer, 2009). Ainsi, l'augmentation du rendement de haricots d'au moins $200 \mathrm{~kg} \mathrm{ha}^{-1}$ pour le témoin à plus de $2.000 \mathrm{~kg} \mathrm{ha}^{-1}$ était observée au Sud-Kivu (CIALCA, 2009). De même, Reij et al., (2003) ont obtenu une augmentation de rendement du sorgho de 200 à $1.700 \mathrm{~kg}$ par hectare due à la combinaison d'engrais minéral et du fumier au Burkina Faso. La restauration de la qualité du sol et la gestion durable des terres, qui doivent se réaliser parallèlement, ne peuvent être résolues par une modification technique mais plutôt à travers l'adoption d'une stratégie entièrement nouvelle qui embrasse tous les aspects du problème et considère tous les constituants d'un développement agricole durable. II faut que la stratégie envisagée prenne en compte des solutions écologiques, alimentaires, économiques et sociales (FAO, 2012). Les pratiques d'intensification écologique peuvent constituer une réponse aux défis des dégradations des ressources naturelles et à la diminution des productions agricoles, en particulier les systèmes de culture en semis direct sous tapis végétale permanente (AFD, 2006; Razafimbelo et al., 2006; FAO, 2012;). Ce système permet la diminution de ruissellement du fait de la présence de couverture morte ou vivante. La forte production de biomasse, issue de la restitution des résidus de cultures laissés en surface, permet un enrichissement en matière organique de l'horizon superficiel du sol (Six et al., 2002). Cette matière organique participe au maintien de la structure du sol et améliore la pénétration de l'eau en profondeur (FAO, 2005; Jiao et al., 2006; Muriel, 2007; Fazle et al., 2010). Ce système permet donc la restauration de la fertilité des sols, et par conséquence, l'amélioration de la production agricole (Razafindramanan et al., 2012). Ainsi, pour accroitre la production, il est important d'appliquer les bonnes pratiques culturales et d'utiliser la variété améliorée (Sallah et al., 2009), car l'amélioration des rendements peut provenir d'un certain nombre de caractéristiques biologiques de la plante ou de pratiques culturelles (Dana, 1978). L'introduction de meilleurs systèmes et technologies agricoles est un enjeu particulièrement important, puisque la meilleure productivité ne signifie pas seulement un volume accru d'aliments, mais aussi une rémunération accrue et une richesse en nutriments des aliments (FAO, 2003). L'objectif de cette étude est d'évaluer différentes variétés de haricots volubiles riches en fer et en zinc diffusées par le projet CIAT/HarvestPlus à l'Est de la RD Congo dans un système intégré de type «jachère-herbage agro-forestière», dans deux différents types de sols. 

micronutriments dans un système intégré d'Agroforesterie sur deux sols contrastés à l'Est de la RD Congo

\section{MATERIEL ET METHODES}

Milieu Expérimental : Les essais ont été conduits dans deux sites situés dans 2 territoires au Sud et au Nord de la province du Sud-Kivu pendant la saison culturale 2016 B. II s'agit du Territoire de Kabare, au Centre de Recherche de l'Institut National pour la Recherche et l'Études Agronomiques à Mulungu (INERA/Mulungu) $\left(02^{\circ}\right.$ 19'907" de latitude Sud, $028^{\circ} 46^{\prime}$ '540" de longitude Est, situé à $1825 \mathrm{~m}$ d'altitude) et dans le «Territoire de Walungu», "groupements» de Mushinga $\left(02^{\circ} 45^{\prime} 592^{\prime}\right.$ " de latitude Sud, $028^{\circ} 39^{\prime} 591^{\prime \prime}$ de longitude Est, situé à 1592 $\mathrm{m}$ d'altitude). Le Sud-Kivu est situé à l'Est de la République Démocratique du Congo. Son relief comprend des montagnes, les chaînes de Mitumba, dont la montagne la plus importante est le sommet de KahuziBiega, $3340 \mathrm{~m}$ d'attitude (DSRP, 2005). Le climat du SudKivu a neuf mois de pluie et trois mois de saison sèche, il s'agit d'un climat tropical humide (Ngongo \& Lunze, 2000). Le Sud- Kivu jouit d'une température moyenne annuelle comprise entre 16 et $20^{\circ} \mathrm{C}$. Le régime pluviométrique est bimodal ; il permet ainsi deux saisons culturales, la première saison $(A)$ s'étalant de miseptembre à mi-novembre et la deuxième $(B)$ de mi-Mars à mi-Juin, suivi d'une courte saison dite $\mathrm{C}$ caractérisée par la culture dans les vallées après drainage de marais pendant la saison sèche (Pypers et al.,2010). La moyenne annuelle des précipitations s'élève à $1572 \mathrm{~mm}$ (Ngongo \& Lunze, 2000). Selon Lunze (2000), les sols de ces 2 sites expérimentaux sont différents. Les sols de Mulungu sont argileux, avec des horizons humifères souvent épais lorsque l'érosion est faible ou absente. Ils sont bien pourvus en matière organique et la teneur en azote total est élevée. Ils ne sont pas acides. Le phosphore ainsi que le potassium peuvent être faible mais pas toujours. Ces sols ont un très bon potentiel de production, mais l'azote devient limitant avec une exploitation continue. Tandis que ceux de Walungu I Mushinga sont très fortement acides avec de forte saturation en aluminium souvent au-delà de $60 \%$. Le phosphore et les bases sont déficients. Le Tableau 1 résumé les caractéristiques de sols de terrains expérimentaux (les échantillons de sols étaient récoltés à la profondeur de 0 à $30 \mathrm{~cm}$ de sol avant l'installation de l'essai).

Tableau 1 : Caractéristiques des sols de sites expérimentaux.

\begin{tabular}{|c|c|c|c|c|c|c|c|c|c|c|c|c|c|}
\hline \multirow[t]{2}{*}{ Site } & \multirow{2}{*}{$\begin{array}{l}\mathrm{pH} \\
\text { eau }\end{array}$} & \multirow[b]{2}{*}{$\begin{array}{l}C \\
(\%)\end{array}$} & \multirow[b]{2}{*}{$\begin{array}{l}N \\
(\%)\end{array}$} & \multirow{2}{*}{$\begin{array}{l}\text { Phosphore } \\
\text { assimilable } \\
\text { (mg P/kg) }\end{array}$} & \multicolumn{6}{|c|}{ Complexe d'échange (méq/100g) } & \multicolumn{3}{|c|}{ Texture } \\
\hline & & & & & $\mathrm{Ca}$ & $\mathrm{Mg}$ & $\mathrm{K}$ & $\begin{array}{l}\mathrm{Fe} \\
(\mathrm{ppm})\end{array}$ & Al+++ & $\mathrm{H}+$ & $\begin{array}{l}\text { Argil } \\
\text { e (\%) }\end{array}$ & $\begin{array}{l}\text { Sable } \\
(\%)\end{array}$ & $\begin{array}{l}\text { Limon } \\
(\%)\end{array}$ \\
\hline Mushinga & 4,31 & 4,09 & 0,42 & 12,43 & 0,31 & 0,65 & 0,09 & 125,5 & 4,40 & 0,70 & 55,79 & 33,70 & 10,51 \\
\hline Mulungu & 5,17 & 2,74 & 0,31 & 20,71 & 2,83 & 0,72 & 0,38 & 122,2 & 1,83 & 0,70 & 63,81 & 20,91 & 15,29 \\
\hline
\end{tabular}

Matériel Végétal : Le matériel végétal utilisé était constitué de 4 variétés de haricots volubiles 'NAMULENGA', 'CODMLV059', 'M211' et 'CUARANTINO 0817 ' à hauts rendements et riches en fer et en zinc. Ces variétés étaient sélectionnées par l'INERA/Mulungu et diffusées par le programme CIAT HarvestPlus à l'Est de la République Démocratique du Congo (RD Congo). Les caractéristiques de ces différentes variétés sont présentées dans le Tableau 2. Trois espèces d'arbustes légumineuses : Leucaena diversifolia, Albizzia chinensis et Calliandra calthyrsus et 3 espèces de graminées: Setaria sphacelata, Pennisetum purpureum et le Tripsacum laxum et une astéracée, le Tithonia diversifolia étaient utilisées dans ces essais.

Tableau 2 : Caractéristiques de différentes variétés de haricots volubiles utilisées dans l'essai.

\begin{tabular}{llcccc}
\hline Variétés & $\begin{array}{c}\text { Couleur de } \\
\text { graines }\end{array}$ & $\begin{array}{c}\text { Nombre de } \\
\text { jours à la } \\
\text { floraison à } \\
\mathbf{5 0 \%}\end{array}$ & $\begin{array}{c}\text { Nombre de jours à } \\
\text { la maturité } \\
\text { physiologique de } \\
\text { gousses à } 50 \%\end{array}$ & $\begin{array}{c}\text { Cycle } \\
\text { végétatif } \\
\text { (jours) }\end{array}$ & $\begin{array}{c}\text { Rendement } \\
\text { moyen } \\
\text { (kg/ha) }\end{array}$ \\
\hline M211 & Blanche & 50 & 103 & 111 & $2.800-4.000$ \\
CUARANTINO 0817 & Blanche pure & 55 & 101 & 111 & $2.500-3.750$ \\
NAMULENGA & $\begin{array}{l}\text { Blanche avec raies } \\
\text { noires }\end{array}$ & 46 & 85 & 92 & $2.700-4.500$ \\
COD MLV059 & Pourpre & 46 & 82 & 106 & $2.500-4.500$ \\
\hline Source : Institut National pour l'Etude et la Recherche Agronomiques (INERA) et CIAT/HarvestPlus (2015)
\end{tabular}


Methodes : Le dispositif expérimental utilisé était le split plot avec comme parcelle principale le type de jachèreherbage agro-forestière (Fig. 1). Elles étaient au total de 11 parcelles dont 9 (T1: Pennisetum purpureum + Leucaena diversifolia-calliandra en haute densité, avec fumier et microdoses d'engrais NPK; T2. Pennisetum purpureum + Leucaena diversifolia-Albizzia chinensis, en haute densité avec fumier et microdoses NPK; T3. Pennisetum purpureum + Leucaena diversifolia Calliandra calothyrsus, en faible densité avec fumier et microdoses NPK ; T4. Pennisetum purpureum + Albizzia chinensis - Leucaena diversifolia, en faible densité avec fumier et microdoses NPK; T5. Setaria sphacelataPennisetum purpureum+ Leucaena diversifolia-Albizzia chinensis en haute densité, avec fumier et microdoses NPK; T6. Setaria sphacelata-Pennisetum purpureum+ Leucaena diversifolia -Calliandra calothyrsus, en haute densité, avec fumier et microdoses NPK; T7. Setaria sphacelata--Pennisetum purpureum +Leucaena diversifolia, en faible densité, avec fumier et microdoses NPK; T8. Setaria sphacelata -Pennisetum purpureum + Leucaena diversifolia-Albizzia chinensis, en faible densité, avec fumier et microdoses NPK; T9. Tithonia diversifolia ou tripsacum -Pennisetum + Leucaena diversifolia -Calliandra calothyrsus, en faible densité, avec fumier et microdoses NPK) issus de la combinaison herbes $x$ arbustes et 2 témoins: T0:recru spontané, avec fumier et microdoses NPK et T00: recru spontané sans fertilisation organo-minérale. La haute densité signifie que les arbustes étaient plantés sur chaque ligne de graminées tandis que, ils étaient plantés sur 3 lignes seulement (2 lignes de bordures et du milieu) pour la faible densité. Chaque parcelle principale était subdivisée en 4 sous parcelles secondaire correspondant aux 4 variétés de haricots volubiles, ce qui a donné le total de 55 sous parcelles de haricots par bloc. L'essai comprenait 5 blocs dans chaque site. La superficie de chaque bloc était de $140 \mathrm{~m} \times 10 \mathrm{~m}$ soit $1400 \mathrm{~m}^{2}$ et la superficie total de l'essai de $140 \mathrm{~m} \times 62 \mathrm{~m}$ soit $8680 \mathrm{~m}^{2}$. La parcelle principale était de $10 \mathrm{~m} \times 10 \mathrm{~m}$ et la parcelle secondaire de $10 \mathrm{~m} \times 2,5 \mathrm{~m}$. Les blocs ou répétitions, perpendiculaires à la pente, étaient séparés par des allées de 3 mètres en tous sens. Une parcelle utile de 10 $\mathrm{m}^{2}$ était mesurée au milieu de la parcelle pour récolter les données sur le rendement soit 3 lignes de haricots contenant un total de 60 plants.
Installation et entretien de l'essai : Le labour était fait partout, le fumier (20 t MS) et les microdoses d'engrais NPK (50 kg/ha) étaient appliquées, sauf au traitement témoin T00. Deux cents $\mathrm{kg}$ de bouse de vache bien décomposée ont été mélangés avec du terreau à la proportion de $1 / 2$, et $500 \mathrm{~g}$ de NPK étaient dissouts dans l'eau en les frottant manuellement dans un sceau jusqu'à la disparition des granules. La suspension était bien agitée lors de l'arrosage du tas du mélange bouseterreau. Ce mélange était appliqué dans la parcelle principale de $100 \mathrm{~m}^{2}$. Les haricots étaient semés aux écartements de $50 \mathrm{~cm}$ entre les lignes et $20 \mathrm{~cm}$ dans la ligne à raison de 2 graines par poquet entre 2 lignes d'herbe à une distance de $15 \mathrm{~cm}$ entre la ligne d'herbe. L'opération de tuteurage est intervenue deux semaines après levée et consistait à fixer une ligne de tuteur entre 2 lignes de haricot, ils étaient espacés de 0,5 $\mathrm{m}$ sur la ligne. Chaque parcelle était traversée de 8 lignes jumellées de Penninsetum en associtation soit avec le Setaria, le Tripsacum ou le Tithonia aux catements de $25 \times 25 \mathrm{~cm}$, ce dernier étant établi aux écartements de $25 \times 50 \mathrm{~cm}$, observant dès lors un dégagement de $25 \mathrm{~cm}$ de part et d'autre. Les graminées et les arbustes étaient plantés avec l'objectif de restaurer et maintenir la fertilité de sols et lutter contre l'érosion car les terrains expérimentaux étaient en pente $(0,8 \%)$. Les graminées étaient coupées chaque deux semaines et servir de paillage entre les lignes de haricots.

Échantillonnage et analyse du sol : Des échantillons composites du sol étaient prélevés par répétition avant l'installation de l'essai, pour les strates de 0 à $30 \mathrm{~cm}$ dans chaque site afin de déterminer les caractéristiques des sites expérimentaux. Les différents échantillons de sols étaient analysés au laboratoire de l'International Institut for Tropical Agriculture (IITA) à Kalambo au Sud-Kivu, RD Congo.

Observation végétative et les maladies : Le paramètre qui a fait l'objet de l'observation végétative était le nombre de nodules mesuré sur 5 plants par parcelle. L'évaluation de nodules était faite durant le stade R6 (à la floraison), lorsque le nombre et le poids des nodules sont généralement les plus importants (Van Schoonhoven, 1992). Les scores (1-9) de différentes maladies étaient obtenus en utilisant les méthodes de van Schoonhoven \& Pastor-Corrales (1992) et allen et al., (1996). 

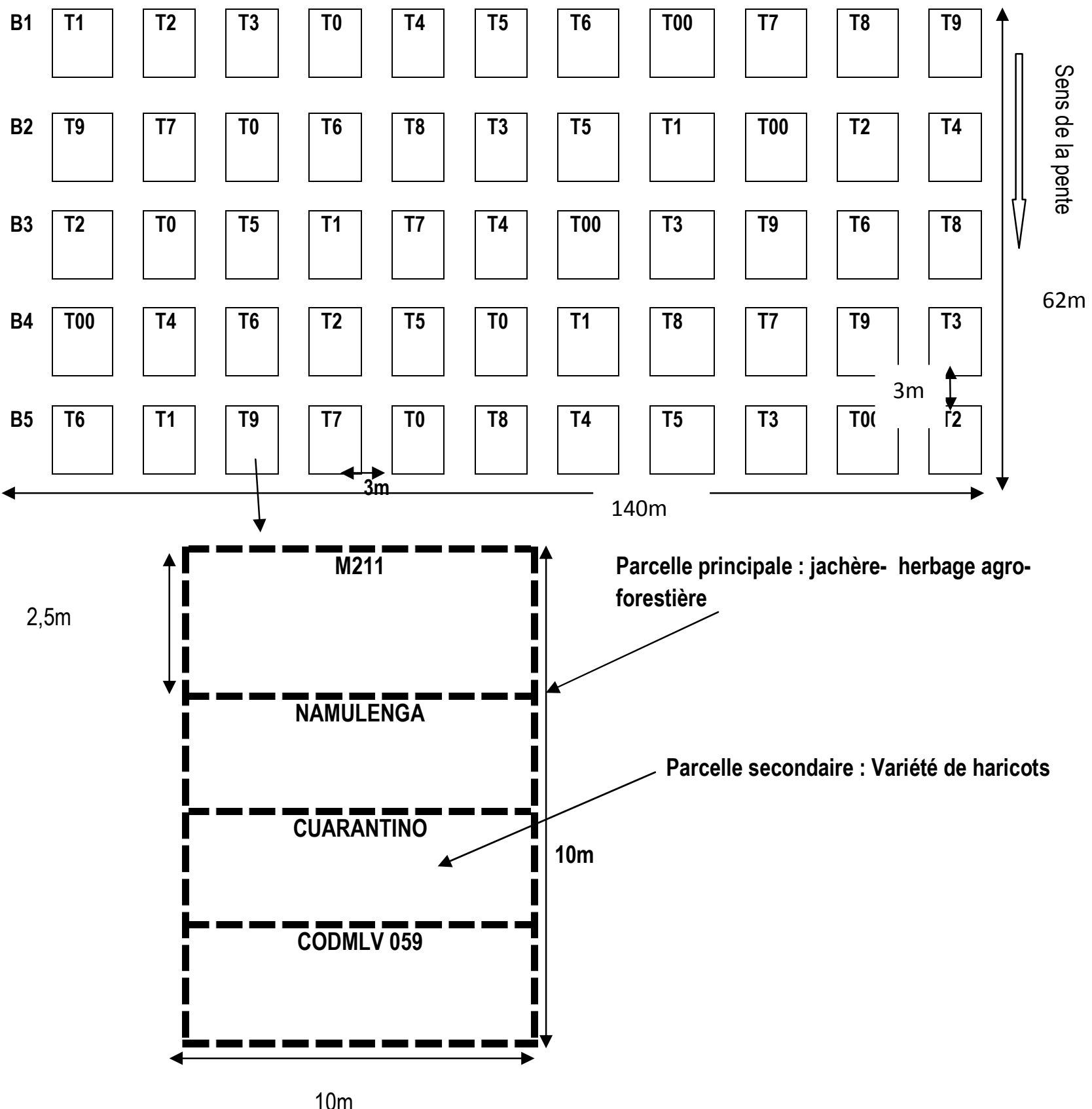

Figure 1 : Schémas du dispositif expérimental

Observations sur les rendements : Pour évaluer les rendements de différentes variétés de haricots, les observations étaient menées sur le nombre de graines par gousse, le poids frais de gousses, le poids sec de 100 graines, le rendement en biomasse par hectare, le rendement en graines par hectare et la teneur en micronutriments ( $\mathrm{Fe}$ et $\mathrm{Zn}$ ) dans les graines de haricots. Le nombre de graines par gousse était obtenu en comptant les graines dans chaque gousse avec un total de 10 gousses récoltées sur 5 plants par traitement. Les poids frais de gousses, le poids de graines et de 100 graines étaient obtenus par pesage par une balance de précision de $3 \mathrm{~kg}$ après séchage de graines au soleil. L'observation sur la biomasse était réalisée au stade de formation de gousse, lorsqu'au moins $50 \%$ de plants avaient formé des gousses. Tous les plants étaient 
récoltés dans une superficie de $1 \mathrm{~m}^{2}$ au milieu de la parcelle utile de chaque traitement, pesés, séchés au soleil et repesés pour réduire l'humidité de graine et permettre une bonne conservation de grains tel que les agriculteurs le font. Le rendement en biomasse par hectare était obtenu par extrapolation du rendement issu de $1 \mathrm{~m}^{2}$ par traitement tandis que, le rendement en graine sec par hectare était obtenu par extrapolation du rendement parcellaire par traitement. Pour ce qui concerne les micronutriments dans les graines de haricots, 20 gousses bien remplis étaient récoltées par

\section{RESULTATS ET DISCUSSION}

Effet de différentes combinaisons d'herbes-arbres sur les rendements, en biomasse de différentes variétés de haricots : II ressort des résultats de l'analyse de la variance que la culture en assiette sous tapis vert formée par la combinaison d'herbes-arbres (ATV), a diminué significativement le rendement en biomasse des haricots de toutes les variétés sur sol relativement fertile à Mulungu en comparaison avec Mushinga (Tableau 3). Les quantités de biomasses produites dans les parcelles avec NPK + Fumier (T0) étaient supérieures à celles de ATV et de parcelles témoins (T00) à Mulungu et ont varié de 967 à 1533; 52 à 462 et 592 à $1048 \mathrm{~kg} / \mathrm{ha}$ pour T0, ATV et T00 respectivement. Aucune différence significative était observée entre les différentes combinaisons d'herbes-arbres pour toutes les variétés et entre les différentes variétés $(p=0,817)$ dans ce site. Contrairement, les biomasses produites dans les parcelles avec la culture en ATV étaient significativement supérieures à celles de témoins $(T 00)(p<0,053)$ pour toutes les variétés à Mushinga. D'une part, les rendements obtenus dans le système de la culture en ATV étaient $9,5,20$ et 8 fois plus élevés que ceux obtenus dans les parcelles témoins n'ayant reçu aucun fertilisant (T00) pour les variétés M211, CUARANTINO, CODMLV059 et NAMULENGA respectivement à Mushinga. D'autre part, les rendements les plus élevés $(582 \mathrm{~kg} / \mathrm{ha}$ et $504 \mathrm{~kg} / \mathrm{ha}$ ) étaient obtenus par la culture ATV + NPK + Fumier avec le traitement T6 et T7 pour les variétés CUARANTINO et NAMULENGA respectivement dans ce site avec un niveau faible de la fertilité de sol (Tableau 3). Les rendements élevés produits par la culture en ATV pourraient être expliqués par le fait qu'en plus du fumier apporté dans les parcelles concernées, il y aurait une décomposition rapide d'une grande quantité de la biomasse de feuilles de Setaria sphacelata et Pennissetum purpureum issus de coupes répétées dans ces parcelles. Ces résultats sont similaires à ceux trouvés par les études antérieures qui confirment la plus grande variété et par traitement et conservées dans une enveloppe en papier pour les analyses. L'analyse a été faite au laboratoire HarvestPlus à Bukavu, RD Congo en respectant la technique de Stangoulis (2010). Pour déterminer les caractéristiques agronomiques des différentes variétés de haricots volubiles, les données étaient analysées avec le logiciel statistique GenStat V. 12 (statistical software VSN International Ltd, 2009) en utilisant l'analyse de la variance. Le Microsoft Office Excel 2007 était utilisé pour tracer les graphiques.

influence de la combinaison des amendements organiques avec les engrais minéraux sur le rendement de haricots en particulier dans les sols pauvres rouges dans la province du Sud-Kivu (CIALCA, 2009). En plus, les quantités de biomasses produites dans les parcelles avec la culture en ATV n'étaient pas significativement différentes entre les différentes parcelles pour toutes les variétés, sauf pour les parcelles T6 qui étaient significativement élevées à ceux de $\mathrm{T} 1$ et $\mathrm{T} 5$ pour la variété CUARANTINO (Tableau 3).

Effet de différentes combinaisons herbes-arbres (culture en ATV) sur les rendements, en graines et en gousses : La culture en assiettes sous tapis vert (ATV) a diminué significativement le rendement en graines et en gousses de haricots dans le sol relativement fertile de Mulungu ( $p=0,001)$. Ces deux rendements étaient significativement inférieurs dans les parcelles de la culture en ATV en comparaison avec ceux des parcelles avec NPK + Fumier (T0) et les parcelles témoins (TO0). Ceci pourrait être expliqué par le fait que les différentes espèces d'herbes se sont développées très rapidement dans ce site, ce qui augmenterait la concurrence en nutriments entre les haricots et ces autres espèces, même si leur coupe était faite régulièrement. Ces deux rendements n'ont pas significativement variés entre les différents traitements de la culture en ATV sur ce type de sol (Tableau 3). Cependant, l'augmentation significative $(p<0.001$ et $p<0.045)$ de rendements en graines et en gousses de haricots par l'application de la combinaison engrais-fumiers sans la culture en ATV étaient obtenus dans les 2 sites avec un effet plus prononcés dans le sol plus pauvre. Mais aussi, les rendements en graines et en gousses de toutes les variétés de haricots obtenues par la culture en ATV+Fumier+NPK étaient significativement plus élevés $(p<0,053)$ que ceux issus de parcelles témoins sans fertilisants dans le site de Mushinga avec un sol plus pauvre en comparaison avec le site de Mulungu avec un sol relativement fertile. L'augmentation 
du rendement de $81,79,81$ et $121 \mathrm{~kg} \mathrm{ha}^{-1}$ pour la culture en ATV+Fumier+NPK et 233, 99, 224 et $176 \mathrm{~kg} \mathrm{ha}^{-1}$ pour le traitement NPK+Fumier par rapport au témoin était respectivement observée pour les variétés M211, CUARANTINO, CODMLV059 et NAMULENGA à Mushinga. Ces résultats corroborent ceux trouvés par Pypers et al., (2010) qui ont montré que les effets des pratiques améliorées sont plus prononcés sur les rendements en grains de haricot sur le sol pauvre que sur sol fertile. Ils ont observé une augmentation de 11 et $24 \%$ sur sol fertile et pauvre respectivement, dans la province du Sud-Kivu en R D Congo. II est également démontré dans la littérature que les sols déficients en azote sont plus susceptibles de répondre mieux à l'application des engrais organiques que ceux ayant une teneur élevée en cet élément (Kaho et al., 2011).

Effet de différentes combinaisons herbes-arbres (ATV) sur le nombre de nodules: Le système de la culture en ATV a augmenté les nombres de nodules dans les deux sites pour toutes les variétés de haricots. Les nombres de nodules les plus bas $(7,9)$ étaient produits dans la parcelle témoin (TO0) par CODMLV059 et le plus élevé $(177,8)$ à Mulungu dans le sol fertile dans la parcelle de T0 pour la variété NAMULENGA. Similairement, le nombre de nodules le plus bas (0) était produit dans la parcelle témoin (T00) et le plus élevé (163 pour la variété $C O M L V 059)$ était observé dans la parcelle en culture en ATV pour le traitement T9 à Mushinga (Tableau 3). Les nombres de nodules produits par la culture en ATV n'étaient pas significativement différents de ceux de T0 pour M211, CODMLV059 et CUARANTINO, sauf pour le traitement T2 pour CUARANTINO. Mais aussi, le nombre de nodule n'a pas significativement varié entre les différentes composantes du système de culture en ATV pour toutes les variétés à Mulungu. Alors qu'à Mushinga sur sol pauvre, le nombre de nodule produit dans le système de culture en ATV pour les traitements T6 et T9 était significativement supérieur à celui de T0 pour la variété COMLV059. La variation de nodules par plant dans les sites expérimentaux, peut être expliquée par la différence de la fertilité de sol dans les deux sites qui influencerait la variation de nodules par plant et par variété. II a était montré par Wouters et al. (1986) que les nodules sont réduits sensiblement dans le sol avec une teneur élevé d'aluminium, c'est le cas de sol de ces deux sites expérimentaux avec de teneur élevée en aluminium échangeable (Lunze, 2000).

Effet de différentes combinaisons herbes-arbres sur les nombres de graines dans les gousses de haricots : En général, la culture en assiettes sous tapis vert (ATV), a augmenté le nombre de graines par gousse de toutes les variétés de haricots dans le sol pauvre de Mushinga en comparaison avec Mulungu. Le nombre moyen de graines par gousse issue de parcelles de culture en ATV était supérieur à celui de parcelles témoins (T00) à Mushinga (Tableau 3). Ce qui peut être du à l'augmentation de fertilisants dans les parcelles en ATV provenance de la décomposition de herbes issus de la coupe répétée de graminées. En plus, le nombre maximal de graines par gousse était relativement plus élevé à Mulungu (6 graines/gousse) comparé à Mushinga (5 graines/gousse).. Cette différence serait due à la différence de caractéristiques de sols observées dans les 2 sites tel que le montre les résultats de l'analyse du sol résumés dans le tableau 1. Le sol de Mushinga contient une concentration en $\mathrm{Al} 3+$ élevé plus que celui de Mulungu, ce qui pourrait réduire l'absorption des éléments nutritifs et de l'eau étant donné que la toxicité de l'aluminium se manifeste par une réduction de développement du système racinaire.

Effet de différentes combinaisons herbes-arbres sur le poids de 100 graines : La combinaison d'herbesarbres a augmenté le poids moyen de 100 graines haricots dans le sol pauvre de Mushinga en comparaison avec Mulungu. Le poids moyen de 100 graines issus de parcelles de culture en ATV était supérieur à celui de parcelles témoins (TOO) à Mushinga sur sol pauvre (Tableau 3). Les graines récoltées à Mulungu étaient plus lourdes que celles de Mushinga. Le poids de 100 graines le plus élevé était de $49 \mathrm{~g}$ à Mulungu et de $46 \mathrm{~g}$ à Mushinga. Cet écart en poids de graines par gousse s'expliquerait par la pauvreté en éléments nutritifs plus prononcée dans le sol de Mushinga par rapport à celui de Mulungu qui peut être dû à la haute concentration en aluminium échangeable du sol et texture différents dans ces 2 sites(Tableau 1). Ces résultats corroborent ceux obtenus par Ashenafi \& Mekuria (2015) qui ont également rapporté des différences entre les poids 100 graines de haricots entre deux sites et entre les variétés en Ethiopie . 


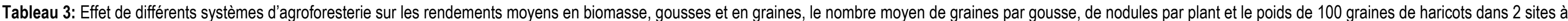
caractéristiques de sols différentes au Sud-Kivu. (V1, V2, V3 et V4 respectivement M211, NAMULENGA, CUARANTINO et COMLV059).

\begin{tabular}{|c|c|c|c|c|c|c|c|c|c|c|c|c|c|c|}
\hline \multirow{2}{*}{$\begin{array}{l}\text { Systèmes de } \\
\text { culture }\end{array}$} & \multirow[b]{2}{*}{ Traitement } & \multirow[b]{2}{*}{ Variétés } & \multicolumn{6}{|l|}{ Mulungu } & \multicolumn{6}{|l|}{ Mushinga } \\
\hline & & & $\begin{array}{l}\text { Biomasse } \\
\text { (kg/ha) }\end{array}$ & Nbre nodules & $\begin{array}{l}\text { Graines par } \\
\text { gousse }\end{array}$ & $\begin{array}{l}\text { Poids } 100 \\
\text { graines }(\mathrm{g})\end{array}$ & $\begin{array}{l}\text { Rdt en gousses } \\
\text { (kg/ha) }\end{array}$ & $\begin{array}{l}\text { Rdt en graines } \\
\text { (kg/ha) }\end{array}$ & Biomasse (kg/ha) & $\begin{array}{l}\text { Nbre } \\
\text { nodules }\end{array}$ & $\begin{array}{l}\text { Graines par } \\
\text { gousse }\end{array}$ & $\begin{array}{l}\text { Poids } 100 \\
\text { raines }(g)\end{array}$ & $\begin{array}{l}\text { Rdt en gousses } \\
\text { (kg/ha) }\end{array}$ & $\begin{array}{l}\text { Rdt en graines } \\
\text { (kg/ha) }\end{array}$ \\
\hline \multirow{5}{*}{ NPK+Fum } & \multirow{4}{*}{$T 0^{*}$} & $\mathrm{~V} 4$ & $967 \mathrm{bc \#}$ & 53,8cdefghi & $4,6 a b c d$ & $49 a$ & $529 \mathrm{e}$ & $304,7 \mathrm{e}$ & $668 \mathrm{a}$ & $93,1 \mathrm{bcdefgh}$ & 4,2abcde & $41 \mathrm{bcd}$ & $352 a$ & $236 a b$ \\
\hline & & V3 & $1282 \mathrm{ab}$ & $111,7 \mathrm{~b}$ & $5,0 \mathrm{abc}$ & $24 \mathrm{kImno}$ & $1545 \mathrm{ab}$ & $1021,2 \mathrm{a}$ & 474 abcdef & $71,3 \mathrm{degh}$ & $4,5 \mathrm{abc}$ & 27hijk & 161defghijkl & 119cdefghijk \\
\hline & & V1 & $1533 a$ & $102,3 \mathrm{bc}$ & $5,7 a b$ & 29ghijk & $1629 a$ & $1144,2 \mathrm{a}$ & $630 \mathrm{ab}$ & 49,4ghik & $4,5 a b c$ & $30 \mathrm{fgh}$ & $339 a b$ & $260 a$ \\
\hline & & V2 & $1150 \mathrm{~b}$ & $177,8 a$ & 4,5abcde & $37 \mathrm{def}$ & $1270 a b c$ & $749,1 \mathrm{~b}$ & 376 cdef & 53,4 fghik & 3,5 cdefg & 29ghi & $306 a b c$ & $201 a b c$ \\
\hline & Moyenne & & 1233 & 111,4 & 4,95 & 34,75 & 1243,25 & 804,8 & 537 & 66,8 & 4,175 & 31,75 & 289,5 & 204 \\
\hline \multirow{5}{*}{ Témoin } & \multirow{4}{*}{ T00 } & V4 & 592 def & $7,9 i$ & $3,5 \mathrm{bcdefg}$ & $48 a$ & 208 ef & $110,5 \mathrm{f}$ & $71 \mathrm{ij}$ & $0 \mathrm{k}$ & $2,8 \mathrm{~g}$ & 28ghij & $18 \mathrm{n}$ & $12 \mathrm{~m}$ \\
\hline & & V3 & $1048 \mathrm{bc}$ & 28,9fghi & $5,3 a b$ & 26ijklmn & $1017 \mathrm{c}$ & $680,8 \mathrm{bc}$ & 116hij & $2,6 \mathrm{ik}$ & $4,5 \mathrm{abc}$ & $24 \mathrm{jklm}$ & $50 \mathrm{klmn}$ & $20 \mathrm{mn}$ \\
\hline & & V1 & 700 cde & $27 \mathrm{fghi}$ & $5,0 a b c$ & 29ghijk & $1236 \mathrm{bcd}$ & $455,2 \mathrm{de}$ & 132 ghij & $0,1 \mathrm{k}$ & $4,1 a b c d e f$ & $211 \mathrm{~m}$ & $37 \mathrm{Imn}$ & $27 \mathrm{mn}$ \\
\hline & & V2 & $818 \mathrm{bcd}$ & $11,8 \mathrm{hi}$ & $4,6 a b c d$ & $34 \mathrm{efg}$ & $931 \mathrm{~d}$ & $553,5 \mathrm{~cd}$ & & Ok & $3 \mathrm{fg}$ & $23 \mathrm{klm}$ & $32 \mathrm{mn}$ & $25 \mathrm{Imn}$ \\
\hline & Moyenne & & 789,5 & 18,9 & 4,6 & 34,25 & 848 & 450 & 91,75 & 0,675 & 3,6 & 24 & 34,25 & 19 \\
\hline \multirow{28}{*}{$\begin{array}{c}\mathrm{NPK}+\mathrm{Fum}+\mathrm{AT} \\
\mathrm{V}\end{array}$} & \multirow{4}{*}{$\mathrm{T} 1$} & V4 & $125 \mathrm{gh}$ & 61,4bcdefghi & $2,4 \mathrm{fg}$ & $37 \mathrm{def}$ & $10 \mathrm{f}$ & $5,5 f$ & 418 bcdef & 93,9acdefgh & 3,3defg & $46 a$ & 63jklmn & $46 \mathrm{klmn}$ \\
\hline & & V3 & $302 \mathrm{fgh}$ & $102,8 \mathrm{bc}$ & 3,9abcdefg & 190 & $O f$ & $0 f$ & 298 efghi & 73,6defgh & 4,1abcdef & $23 \mathrm{klm}$ & 74ijklmn & $54 \mathrm{jklmn}$ \\
\hline & & V1 & $293 \mathrm{fgh}$ & $92,5 \mathrm{bcde}$ & 3,8abcdefg & $24 \mathrm{k} \mid \mathrm{mno}$ & of & $0 f$ & 462 abcdef & 62,2 efghi & $4,2 a b c d e$ & 28 ghij & 167defghijk & 118cdefghijk \\
\hline & & V2 & $257 \mathrm{fgh}$ & $82,7 \mathrm{bcdefg}$ & 3,5bcdefg & 31ghi & $50 \mathrm{f}$ & $28,5 f$ & 368 cdefg & 56,9 gh hik & $4,4 a b c d$ & $30 \mathrm{fgh}$ & 166defghijk & 108defghijkl \\
\hline & \multirow{5}{*}{ T2 } & V4 & $183 \mathrm{gh}$ & $81,4 \mathrm{bcdefg}$ & $2,4 \mathrm{fg}$ & $39 \mathrm{cde}$ & $28 f$ & $15,4 \mathrm{f}$ & $570 \mathrm{abc}$ & 108abcdefg & $3,1 \mathrm{efg}$ & $42 a b c$ & 141fghijklmn & 96fghijklmn \\
\hline & & V3 & $252 \mathrm{fgh}$ & 54,7cdefghi & 3,8abcdefg & 190 & of & of & $380 \mathrm{cdef}$ & 112adcdef & $4,4 a b c d$ & 24jklm & $217 \mathrm{bcdefgh}$ & 136cdefghij \\
\hline & & V1 & $240 \mathrm{fgh}$ & 48,5cdefghi & $3,4 \mathrm{bcdefg}$ & $24 \mathrm{klmno}$ & of & $0 f$ & 536 abcde & 53,7fghik & 4,2abcde & 29ghi & 164defghijk & 118cdefghijk \\
\hline & & V2 & $189 \mathrm{gh}$ & $78,7 \mathrm{bcdefg}$ & 3,7abcdefg & $24 \mathrm{klmno}$ & $29 f$ & $18,3 \mathrm{f}$ & 484 abcdef & $93,4 \mathrm{bcdefgh}$ & 3,2efg & 28ghij & 280abcde & 184abcde \\
\hline & & V4 & $178 \mathrm{gh}$ & $97,8 \mathrm{bcdfg}$ & $2,0 \mathrm{~g}$ & $40 \mathrm{bcd}$ & $27 f$ & $14,7 f$ & 370 cdefg & $79,9 \mathrm{cdefgh}$ & 3,9abcdefg & $34 \mathrm{ef}$ & 150fghijklm & 105efghijklm \\
\hline & \multirow{3}{*}{ T3 } & V3 & $280 \mathrm{fgh}$ & $71 \mathrm{bcdefg}$ & $3,4 b c d e f g$ & $22 \mathrm{mno}$ & $9 f$ & $4,9 f$ & 382 cdef & 70defgh & 3,3defg & 24jklm & 249abcdef & 173abcdef \\
\hline & & V1 & 395 efgh & $66,7 \mathrm{bcdefgh}$ & 2,7 defg & 26ijklmn & of & of & 504 abcdef & 72,2 defgh & 3,8abcdefg & 29ghi & 178defghij & 123cdefghijk \\
\hline & & V2 & $212 \mathrm{gh}$ & $111,5 b$ & 3,1 cdefg & 30ghij & $95 f$ & $54,4 \mathrm{f}$ & 330 defgh & 60,8 efghik & 3,6bcdefg & 27hijk & $224 b c d e f g$ & 149bcdefgh \\
\hline & \multirow{4}{*}{ T4 } & V4 & 388 efgh & $71,4 \mathrm{bcdefg}$ & 3,1 cdefg & $41 \mathrm{bcd}$ & $8 f$ & $2,9 f$ & 458 abcdf & $128 a b c d$ & 3,2efg & $38 \mathrm{cde}$ & 132fghijklmn & 9ofghijklmn \\
\hline & & V3 & 358 efgh & $62,9 \mathrm{bcdefghi}$ & $5,3 a b c$ & 21 no & of & of & 378 cdef & $125 a b c d$ & $4,1 \mathrm{abcdef}$ & $20 \mathrm{~m}$ & 67jkkmn & $47 \mathrm{klmn}$ \\
\hline & & V1 & 462 defg & $76,1 \mathrm{bcdefg}$ & 3,3bcdefg & $27 \mathrm{hijklm}$ & $5 f$ & $2,6 f$ & 530 abcde & 68,9 defgh & $4,7 a b$ & 29ghi & 156efghijklm & 110defghijkl \\
\hline & & V2 & 350 efgh & $76,5 \mathrm{bcdefg}$ & 4, 1abcdef & 30ghij & $109 f$ & $51,2 f$ & 494 abcdef & 82,9 cdefgh & 3,9abcdefg & 30fgh & 195cdefghi & 130cdefghijk \\
\hline & \multirow{4}{*}{ T5 } & V4 & $52 \mathrm{~h}$ & $57,7 \mathrm{bcdefghi}$ & $2,4 \mathrm{fg}$ & $45 a b$ & $15 f$ & $7,8 \mathrm{f}$ & 556 abcd & 88,6cdefgh & 3,6bcdefg & $37 \mathrm{de}$ & 67jklmn & $49 j k 1 m n$ \\
\hline & & V3 & $150 \mathrm{gh}$ & $65,3 \mathrm{bcdefgh}$ & 2,6 defg & $231 \mathrm{mno}$ & $16 f$ & $9 f$ & 272 fghij & $96,2 \mathrm{bcdefgh}$ & $4,4 a b c d$ & $23 \mathrm{klm}$ & 81ijklmn & 59ijklmn \\
\hline & & V1 & $125 \mathrm{gh}$ & $71,7 \mathrm{bcdefg}$ & $2,3 \mathrm{fg}$ & 29ghijk & $10 f$ & $5,8 \mathrm{f}$ & 374 cdef & 44,3hik & $4,5 \mathrm{abcc}$ & 28ghij & 120ghijklmn & 84ghijklmn \\
\hline & & V2 & 338 efgh & $93,6 \mathrm{bcdf}$ & 3,1 cdefg & 32fgh & $68 f$ & $36,3 \mathrm{f}$ & 329defgh & 52fghik & 3,3defg & $30 \mathrm{fgh}$ & 152fghijklm & 98efghijklmn \\
\hline & \multirow{4}{*}{ T6 } & V4 & 346 efgh & 37,2efghi & 3,3bcdefg & $44 a b c$ & $15 f$ & $7,6 \mathrm{f}$ & 421 bcdef & $162 a$ & $3,6 \mathrm{bcefg}$ & $39 \mathrm{bcd}$ & 130fghijklmn & 88fghijklmn \\
\hline & & V3 & $269 \mathrm{fgh}$ & $66,9 \mathrm{bcdefgh}$ & $3,6 \mathrm{bcdefg}$ & $25 \mathrm{jklmn}$ & $13 f$ & $7,6 \mathrm{f}$ & $582 \mathrm{abc}$ & $139 a b c$ & 4,4abcd & 24jklm & 212 cdefgh & 129cdefghijk \\
\hline & & V1 & $212 \mathrm{gh}$ & 51,1 cdefghi & 2,5efg & $27 \mathrm{hijklm}$ & $18 \mathrm{f}$ & $9,9 f$ & 422 bcdef & 82,4cdefgh & 4,4abcd & 29ghi & 196cdefghi & 135cdefghij \\
\hline & & V2 & $255 \mathrm{fgh}$ & $64,7 \mathrm{bcdefgh}$ & $3,5 \mathrm{bcdefg}$ & 28hijkl & $51 \mathrm{f}$ & $28,2 f$ & 410 bcdef & $113 a b c d e f$ & 3,5 cdefg & $32 \mathrm{fg}$ & 284abcd & 194abcd \\
\hline & \multirow{4}{*}{ T7 } & V4 & $109 \mathrm{gh}$ & 44,7defghi & $2,4 f g$ & $41 \mathrm{bcd}$ & $4 f$ & $2 f$ & 300 efghi & 103abcdefgh & 3,2efg & $43 a b$ & 97hijklmn & 73hijklmn \\
\hline & & V3 & $225 \mathrm{gh}$ & $68,2 \mathrm{bcdefg}$ & 3,6bcdefg & 21 no & of & $0 \mathrm{f}$ & 392 bcdef & 79,7 cdefgh & $4,6 a b c$ & 25ijkl & 109ghijklmn & 89fghijklmn \\
\hline & & V1 & $252 \mathrm{fgh}$ & $57,6 \mathrm{bcdefghi}$ & 3,6bcdefg & 26ijklmn & of & $0 f$ & 475 abcdef & 67defgh & 4,2abcde & 27hijk & 122ghijklmn & 85ghijklmn \\
\hline & & V2 & $270 \mathrm{fgh}$ & $71,6 \mathrm{bcdefg}$ & 2,7 defg & 31ghi & $22 f$ & $10,2 f$ & 504 abcdef & $96,8 \mathrm{bcdefgh}$ & $4 a b c d e f$ & $32 \mathrm{fg}$ & 253abcdef & $167 \mathrm{bcdefg}$ \\
\hline
\end{tabular}




\begin{tabular}{|c|c|c|c|c|c|c|c|c|c|c|c|c|c|}
\hline \multirow{4}{*}{ T8 } & V4 & $165 \mathrm{gh}$ & $64,8 \mathrm{bcdefgh}$ & $2,1 \mathrm{fg}$ & $47 a$ & $4 f$ & $0,9 f$ & 488 abcdef & $151 a b$ & 3,5 cdefg & $42 a b c$ & $211 \mathrm{cdefgh}$ & 145bcdefghi \\
\hline & V3 & $245 \mathrm{fgh}$ & $65,5 \mathrm{bcdefgh}$ & 3,8abcdefg & $24 \mathrm{k} / \mathrm{mno}$ & $6 f$ & $2,9 \mathrm{f}$ & 534 abcde & 121abcde & $4,6 a b c$ & 27hijk & 161defghijkl & 112defghijkl \\
\hline & V1 & $220 \mathrm{gh}$ & 49,1cdefghi & 3,3bcdefg & 29ghijk & $3 f$ & $2 f$ & 380 cdef & 57,8 fghik & 3,9abcdefg & 29ghi & 145fghijklm & 101efghijklm \\
\hline & V2 & 367 efgh & $67,3 \mathrm{bcdefgh}$ & 3,8abcdefg & 30ghij & $44 f$ & $26,2 f$ & 298 efghi & 67,6 defgh & 4,1abcdef & 30fgh & 193cdefghi & 129cdefghijk \\
\hline \multirow{4}{*}{ T9 } & V4 & $158 \mathrm{gh}$ & $64,8 \mathrm{bcdefgh}$ & $3,3 \mathrm{bcdefg}$ & $45 a b$ & $3 f$ & $1,7 f$ & $576 a b c$ & $163 a$ & $2,8 \mathrm{~g}$ & $37 \mathrm{de}$ & 153fghijklm & 102efghijklm \\
\hline & V3 & $315 \mathrm{fgh}$ & $90,3 \mathrm{bcdefg}$ & $4,6 \mathrm{abcd}$ & $22 \mathrm{mno}$ & $5 f$ & $2,6 \mathrm{f}$ & $398 \mathrm{bcdef}$ & 120abcde & 4abcdef & $25 \mathrm{jjk} \mid$ & 132fghijklmn & 91fghijklmn \\
\hline & V1 & $250 \mathrm{fgh}$ & $83,5 \mathrm{bcdefg}$ & 2,6defg & 29ghijk & $4 f$ & $2,3 \mathrm{f}$ & 440 abcdef & 60,2efghik & $4,8 \mathrm{a}$ & 29 ghi & 144fghijklm & 101efghijklm \\
\hline & V2 & $295 \mathrm{fgh}$ & $64,9 \mathrm{bcdefgh}$ & $4,6 a b c d$ & 30ghij & $36 f$ & $20,1 \mathrm{f}$ & 442 abcdef & 57, ,fghik & 4,2abcde & $31 \mathrm{fgh}$ & 212 cdefgh & 156bcdefgh \\
\hline Moyenne & & 252,28 & 70,48 & 3,27 & 30,06 & 19,64 & 10,60 & 432,08 & 90,66 & 3,92 & 30,56 & 161,03 & 110,39 \\
\hline & LSD & 364.9 & 55.80 & 2,0 & 5.2 & 364 & 188 & 238.3 & 61 & 1,1 & 4,1 & 125 & 87 \\
\hline & Fpr. & 0.817 & 0.375 & 0,01 & 0.337 & 0.001 & 0.001 & 0.056 & 0,49 & 0.001 & 0.001 & 0.050 & 0.096 \\
\hline
\end{tabular}
\#: Les chiffres suivis par une même lettre dans la même colonne ne sont pas significativement différents selon le test de LSD (la plus petite différence significative) $p<0,05$.

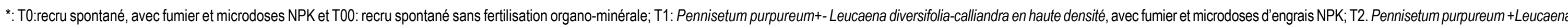

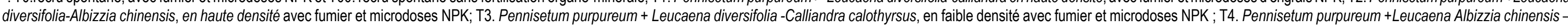

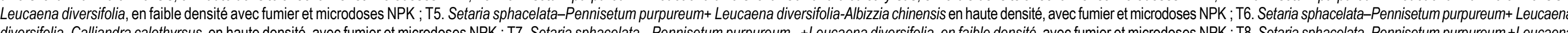
diversifolia-Albizzia chinensis, en frible densité, avec fumier et microdoses NPK. T9. Tithonia diversiflia ou tripsacum -Pennisetum + Leucaena diversifolia -Calliandra calothyrsus, en faible densité, avec fumier et microdoses NPK 
Ntamwira et al., J. Appl. Biosci. 2017 Évaluation agronomique des variétés de haricot volubile riches en micronutriments dans un système intégré d'Agroforesterie sur deux sols contrastés à l'Est de la RD Congo

Tableau 4: Effet de différents systèmes intégrés d'agroforesterie sur les maladies de haricots dans 2 types de sols avec caractéristiques différents au Sud-Kivu

\begin{tabular}{|c|c|c|c|c|c|c|c|c|}
\hline \multirow{2}{*}{$\begin{array}{l}\text { Système de } \\
\text { culture }\end{array}$} & \multirow[b]{2}{*}{ Traitement } & \multirow[b]{2}{*}{ Variétés } & \multicolumn{3}{|c|}{ Mulungu } & \multicolumn{3}{|c|}{ Mushinga } \\
\hline & & & $\begin{array}{l}\text { Taches } \\
\text { anguleuses }\end{array}$ & Bactérie & Virus & $\begin{array}{l}\text { Taches } \\
\text { anguleuses }\end{array}$ & Bactérie & Virus \\
\hline \multirow{5}{*}{ NPK+FUM } & \multirow{5}{*}{$\mathrm{TO}^{*}$} & CODMLV059 & $2 b$ & $1 a$ & $2 a b$ & $3 b$ & $1 a$ & $2 b$ \\
\hline & & CUARANTINO & $3 a b$ & $1 a$ & $2 a b$ & $3 b$ & $1 \mathrm{a}$ & $1 c$ \\
\hline & & M211 & $3 a b$ & $1 \mathrm{a}$ & $2 a b$ & $3 b$ & $1 \mathrm{a}$ & $1 c$ \\
\hline & & NAMULENGA & $2 b$ & $1 a$ & $2 a b$ & $3 b$ & $1 a$ & $1 \mathrm{c}$ \\
\hline & & Moyenne & 3 & 1 & 2 & 3 & 1 & 1 \\
\hline \multirow{5}{*}{ Témoin } & \multirow{5}{*}{ T00 } & CODMLV059 & $3 a b$ & $1 a$ & $2 a b$ & $3 b$ & $1 a$ & $3 a$ \\
\hline & & CUARANTINO & $4 a$ & $1 a$ & $3 a$ & $3 b$ & $1 a$ & $3 a$ \\
\hline & & M211 & $4 a$ & $1 \mathrm{a}$ & $3 a$ & $3 b$ & $1 \mathrm{a}$ & $2 b$ \\
\hline & & NAMULENGA & $3 a b$ & $1 a$ & $2 a b$ & $2 b$ & $1 a$ & $2 b$ \\
\hline & & Moyenne & 4 & 1 & 3 & 3 & 1 & 3 \\
\hline \multirow{39}{*}{$\begin{array}{l}\text { NPK+FUM } \\
+ \text { ATV }\end{array}$} & \multirow{4}{*}{$\mathrm{T} 1$} & CODMLV059 & $3 a b$ & $1 a$ & $2 a b$ & $3 b$ & $1 a$ & $1 c$ \\
\hline & & CUARANTINO & $3 a b$ & $1 a$ & $1 b$ & $3 b$ & $1 \mathrm{a}$ & $2 b$ \\
\hline & & M211 & $4 a$ & $1 \mathrm{a}$ & $1 b$ & $2 b$ & $1 \mathrm{a}$ & $2 b$ \\
\hline & & NAMULENGA & $3 a b$ & $1 \mathrm{a}$ & $2 a b$ & $3 b$ & $1 \mathrm{a}$ & $2 b$ \\
\hline & \multirow{4}{*}{$\mathrm{T} 2$} & CODMLV059 & $3 a b$ & $1 a$ & $2 a b$ & $3 b$ & $1 a$ & $1 c$ \\
\hline & & CUARANTINO & $3 a b$ & $1 \mathrm{a}$ & $1 b$ & $3 b$ & $1 \mathrm{a}$ & $1 c$ \\
\hline & & M211 & $4 a$ & $1 a$ & $1 b$ & $3 b$ & $1 \mathrm{a}$ & $2 b$ \\
\hline & & NAMULENGA & $4 a$ & $1 \mathrm{a}$ & $2 a b$ & $4 a$ & $1 \mathrm{a}$ & $1 c$ \\
\hline & \multirow{4}{*}{ T3 } & CODMLV059 & $3 a b$ & $1 a$ & $1 b$ & $3 b$ & $1 \mathrm{a}$ & $1 \mathrm{c}$ \\
\hline & & CUARANTINO & $3 a b$ & $1 \mathrm{a}$ & $1 b$ & $3 b$ & $1 \mathrm{a}$ & $1 c$ \\
\hline & & M211 & $4 a$ & $1 a$ & $1 b$ & $3 b$ & $1 \mathrm{a}$ & $2 b$ \\
\hline & & NAMULENGA & $3 a b$ & $1 a$ & $1 b$ & $3 b$ & $1 a$ & $2 b$ \\
\hline & \multirow{4}{*}{$\mathrm{T} 4$} & CODMLV059 & $3 a b$ & $1 \mathrm{a}$ & $1 b$ & $3 b$ & $1 a$ & $2 b$ \\
\hline & & CUARANTINO & $3 a b$ & $1 a$ & $1 b$ & $3 b$ & $1 a$ & $1 c$ \\
\hline & & M211 & $4 a$ & $1 \mathrm{a}$ & $1 b$ & $3 b$ & $1 \mathrm{a}$ & $2 b$ \\
\hline & & NAMULENGA & $4 a$ & $1 a$ & $2 a b$ & $3 b$ & $1 a$ & $2 b$ \\
\hline & \multirow{4}{*}{ T5 } & CODMLV059 & $3 a b$ & $1 \mathrm{a}$ & $2 a b$ & $3 b$ & $1 \mathrm{a}$ & $2 b$ \\
\hline & & CUARANTINO & $3 a b$ & $1 a$ & $1 b$ & $3 b$ & $1 a$ & $2 b$ \\
\hline & & M211 & $4 a$ & $1 \mathrm{a}$ & $1 b$ & $3 b$ & $1 \mathrm{a}$ & $2 b$ \\
\hline & & NAMULENGA & $4 a$ & $1 \mathrm{a}$ & $1 b$ & $3 b$ & $1 \mathrm{a}$ & $2 b$ \\
\hline & \multirow{4}{*}{ T6 } & CODMLV059 & $3 a b$ & $1 a$ & $2 a b$ & $3 b$ & $1 a$ & $1 c$ \\
\hline & & CUARANTINO & $3 a b$ & $1 \mathrm{a}$ & $1 b$ & $3 b$ & $1 a$ & $2 b$ \\
\hline & & M211 & $4 a$ & $1 a$ & $1 b$ & $3 b$ & $1 a$ & $2 b$ \\
\hline & & NAMULENGA & $4 a$ & $1 \mathrm{a}$ & $2 a b$ & $3 b$ & $1 \mathrm{a}$ & $1 c$ \\
\hline & \multirow{4}{*}{$\mathrm{T} 7$} & CODMLV059 & $3 a b$ & $1 \mathrm{a}$ & $1 b$ & $3 b$ & $1 \mathrm{a}$ & $2 b$ \\
\hline & & CUARANTINO & $3 a b$ & $1 \mathrm{a}$ & $1 b$ & $3 b$ & $1 a$ & $1 c$ \\
\hline & & M211 & $4 a$ & $1 a$ & $1 b$ & $3 b$ & $1 a$ & $2 b$ \\
\hline & & NAMULENGA & $4 a$ & $1 a$ & $1 b$ & $3 b$ & $1 a$ & $1 c$ \\
\hline & \multirow{3}{*}{ T8 } & CODMLV059 & $3 a b$ & $1 a$ & $1 b$ & $3 b$ & $1 a$ & $1 c$ \\
\hline & & CUARANTINO & $4 a$ & $1 a$ & $1 b$ & $3 b$ & $1 a$ & $2 b$ \\
\hline & & M211 & $4 a$ & $1 \mathrm{a}$ & $2 a b$ & $3 b$ & $1 a$ & $2 b$ \\
\hline & \multirow{8}{*}{ T9 } & NAMULENGA & $4 a$ & $1 a$ & $2 a b$ & $3 b$ & $1 a$ & $2 b$ \\
\hline & & CODMLV059 & $3 a b$ & $1 \mathrm{a}$ & $1 b$ & $3 b$ & $1 a$ & $2 b$ \\
\hline & & CUARANTINO & $4 a$ & $1 \mathrm{a}$ & $1 b$ & $3 b$ & $1 a$ & $2 b$ \\
\hline & & M211 & $4 a$ & $1 \mathrm{a}$ & $1 b$ & $3 b$ & $1 a$ & $2 b$ \\
\hline & & NAMULENGA & $4 a$ & $1 a$ & $1 b$ & $3 b$ & $1 a$ & $2 b$ \\
\hline & & Moyenne & 4 & 1 & 1 & 3 & 1 & 2 \\
\hline & & LSD & 1 & 0.1 & 1 & 0,8 & 0.1 & 0.5 \\
\hline & & Fpr. & 0.38 & 0.53 & 0.18 & 0.25 & 0.12 & 0.02 \\
\hline
\end{tabular}


Effet de différentes combinaisons herbes-arbres sur les maladies des haricots: Le score moyen de la maladie de tache anguleuse de haricots n'était pas significativement différent entre tous les traitements pour toutes les variétés étudiées et entre les variétés dans les 2 sites. Ce qui laisse à croire que les différentes variétés ont manifesté une tolérance égale à cette maladie. Le score a varié de 3 à 4 à Mulungu et de 2 à 3 à Mushinga pour les différents traitements dans le système de culture en ATV et T00 alors qu'une variation allant de 2 à 3 était observée à Mulungu et le score était de 3 à Mushinga pour T0 (Tableau 4). L'échelle des scores de référence pour l'évaluation des maladies était de 1 à 9 . La même tendance a était observée à Mulungu pour le score de la maladie virale, il n'a pas significativement varié entre les différents traitements et entre les variétés. Contrairement au site Mulungu, le score de la maladie virale a varié selon les traitements sauf $\mathrm{T} 5$ et $\mathrm{T} 9$ et les variétés dans le site de Mushinga . Le score le plus bas (1) était observé dans les parcelles où les fertilisants étaient appliqués (ATV et T0) et le plus élevé (3) dans les parcelles témoins sans fertilisants. Ces résultats confirment les observations antérieures qui ont montré que les plants vigoureux résistent plus aux maladies que les chétifs. C'est le cas des observations menées sur le riz par Imrani et al. (2014) qui ont montré que le potassium augmenterait la vigueur de la plante et la rend plus résistante contre la maladie. Par ailleurs, les variétés ont affiché une réaction résistante (1 à 3) à la maladie virale dans le 2 sites (Tableau 4). Cependant, les plants n'ont pas présentés des symptômes des bactéries. Le score de la maladie était de 1 pour tous les traitements et variétés dans les 2 sites.

Effet de différentes combinaisons herbes-arbres sur la teneur en fer et zinc dans les graines de haricots : La teneur moyenne en fer et en zinc dans les graines de haricots produits dans le système de culture en ATV était inférieur à ceux produit dans les parcelles avec NPK+Fuimier (T0) et le témoin T00 dans les 2 sites sauf pour le fer à Mushinga où la teneur en cet élément était plus élevé dans les traitements de culture en ATV en comparaison avec le témoin T00. La quantité de fer a varié entre 89 et 53 et de 93 à 51 ppm à Mulungu et Mushinga respectivement. Tandis que le zinc a varié de 23 à 41 et de 24 à 47 ppm à Mulungu et Mushinga respectivement. La variété CUARANTINO contenait la plus grande quantité de fer et de zinc dans les 2 sites, elles ont varié de 62 à 89 ppm, 24 et $41 ; 71$ et 93 , 45 et 40 pour le fer et zinc à Mulungu et Mushinga respectivement (Tableau 5). Ces résultats montrent que la teneur en différents éléments nutritifs dans les graines de haricots varierait de mode de gestion d'un sol et d'une variété à l'autre. Lestienne (2004) a montré également l'influence de génotype et les facteurs environnementaux sur la teneur en de nombreux nutriments dans les grains de mil au Nigeria. 
Ntamwira et al., J. Appl. Biosci. 2017 Évaluation agronomique des variétés de haricot volubile riches en micronutriments dans un système intégré d'Agroforesterie sur deux sols contrastés à l'Est de la RD Congo

Tableau 5 : Effet des différents traitements sur le taux en fer et zinc dans les graines de haricots dans 2 sites avec caractéristiques de sols différents.

\begin{tabular}{|c|c|c|c|c|c|c|}
\hline \multirow{2}{*}{$\begin{array}{l}\text { Système de } \\
\text { culture }\end{array}$} & \multirow{2}{*}{ Traitements } & \multirow[b]{2}{*}{ Variétés } & \multicolumn{2}{|c|}{ Mulungu } & \multicolumn{2}{|c|}{ Mushinga } \\
\hline & & & Fer (ppm) & Zinc (ppm) & Fer (ppm) & Zinc (ppm) \\
\hline \multirow{5}{*}{ NPK+FUM } & \multirow{4}{*}{$\mathrm{TO}^{*}$} & CURANTINO & 83 & 33 & 84 & 47 \\
\hline & & NAMULENGA & 81 & 39 & 71 & 40 \\
\hline & & M211 & 75 & 31 & 78 & 45 \\
\hline & & COMLV059 & 80 & 27 & 76 & 37 \\
\hline & Moyenne & & 80 & 33 & 77 & 42 \\
\hline \multirow{5}{*}{ Témoin } & \multirow{4}{*}{ T00 } & CURANTINO & 86 & 40 & 77 & 46 \\
\hline & & NAMULENGA & 66 & 24 & 73 & 35 \\
\hline & & M211 & 57 & 27 & 54 & 40 \\
\hline & & COMLV059 & 89 & 36 & 72 & 38 \\
\hline & \multirow[t]{2}{*}{ Moyenne } & & 74 & 32 & 69 & 40 \\
\hline \multirow{37}{*}{$\begin{array}{l}\text { NPK+FUM } \\
+ \text { ATV }\end{array}$} & & CURANTINO & ND & ND & 84 & 47 \\
\hline & \multirow{3}{*}{$\mathrm{T} 1$} & NAMULENGA & 69 & 25 & 51 & 28 \\
\hline & & M211 & ND & ND & 63 & 33 \\
\hline & & COMLV059 & ND & ND & 77 & 33 \\
\hline & & CURANTINO & 89 & 37 & 74 & 41 \\
\hline & \multirow{4}{*}{ T2 } & NAMULENGA & 65 & 27 & 72 & 36 \\
\hline & & M211 & ND & ND & 67 & 38 \\
\hline & & COMLV059 & ND & ND & 77 & 35 \\
\hline & & CURANTINO & 73 & 41 & 75 & 42 \\
\hline & \multirow{4}{*}{ T3 } & NAMULENGA & 62 & 25 & 58 & 29 \\
\hline & & M211 & 64 & 27 & 67 & 36 \\
\hline & & COMLV059 & 69 & 29 & 70 & 35 \\
\hline & & CURANTINO & 68 & 37 & 74 & 39 \\
\hline & \multirow{4}{*}{$\mathrm{T} 4$} & NAMULENGA & 72 & 30 & 66 & 32 \\
\hline & & M211 & 53 & 29 & 71 & 40 \\
\hline & & COMLV059 & 65 & 30 & 65 & 32 \\
\hline & & CURANTINO & ND & ND & 88 & 43 \\
\hline & \multirow{3}{*}{ T5 } & NAMULENGA & 66 & 23 & 68 & 34 \\
\hline & & M211 & ND & ND & 69 & 38 \\
\hline & & COMLV059 & 54 & 28 & 84 & 40 \\
\hline & \multirow{5}{*}{ T6 } & CURANTINO & ND & ND & 93 & 45 \\
\hline & & NAMULENGA & 86 & 33 & 72 & 32 \\
\hline & & M211 & 65 & 27 & 81 & 39 \\
\hline & & COMLV059 & 80 & 27 & 86 & 38 \\
\hline & & CURANTINO & ND & ND & 71 & 40 \\
\hline & \multirow{3}{*}{$\mathrm{T} 7$} & NAMULENGA & 75 & 27 & 59 & 31 \\
\hline & & M211 & ND & ND & 63 & 31 \\
\hline & & COMLV059 & ND & ND & 51 & 24 \\
\hline & \multirow{4}{*}{ T8 } & CURANTINO & 67 & 31 & 89 & 47 \\
\hline & & NAMULENGA & 80 & 32 & 61 & 34 \\
\hline & & M211 & 62 & 30 & 73 & 42 \\
\hline & & COMLV059 & 65 & 25 & 77 & 37 \\
\hline & \multirow{4}{*}{ T9 } & CURANTINO & 62 & 24 & 80 & 46 \\
\hline & & NAMULENGA & 71 & 29 & 68 & 37 \\
\hline & & M211 & ND & ND & 58 & 31 \\
\hline & & COMLV059 & 70 & 35 & 61 & 33 \\
\hline & Moyenne & & 69 & 30 & 71 & 37 \\
\hline
\end{tabular}

ND : Non disponible 
Relation entre les différents paramètres du rendement dans deux sites à caractéristiques de sols différentes : L'analyse de la corrélation a montré qu'il n'y avait pas une relation significative entre le nombre de nodules, rendement en biomasses, en gousses et en grains dans le site de Mulungu (Tableau 6). Cependant, des relations significativement positives $(P<0,001)$ étaient observées entre le nombre de nodules et le rendement en biomasse et entre le rendement en biomasses, en gousses et en grains dans le site de Mushinga (Tableau 6). Ce qui pourrait être expliqué par le fait que la variété qui produit beaucoup de nodules donnerait aussi un rendement plus élevé en graines et en gousses. Ces résultats sont similaires à ceux obtenus par Sana (2011) qui a observé une relation significative entre les paramètres de croissance, ceux de nodulations ainsi que les productions de graines du sulla en Tunisie.

Tableau 6 : Relation entre les différents paramètres du rendement dans deux sites à caractéristiques du sol différentes

\begin{tabular}{llllll}
\hline Site & Paramètres & $\begin{array}{l}\text { Rendement en } \\
\text { biomasse }\end{array}$ & Nodules & $\begin{array}{l}\text { Rendement en } \\
\text { graines }\end{array}$ & $\begin{array}{l}\text { Rendement } \\
\text { gousses }\end{array}$ \\
\hline Mushinga & Rendement en biomasse & 1 & & & \\
& Nodules & $0,599^{* *}$ & 1 & & \\
& Rendement en graines & $0,619^{* *}$ & 0,224 & 1 & 1 \\
& Rendement gousses & $0,598^{* *}$ & 0,244 & $0,991^{* *}$ & 1 \\
\hline Mulungu & Rendement en biomasse & 1 & & & \\
& Nodules & 0,184 & 1 & & 1 \\
& Rendement en graines & $0,946^{* *}$ & 0,219 & 1 & 1 \\
& Rendement en gousses & $0,928^{* *}$ & 0,172 & $0,981^{* *}$ & 1 \\
\hline ** Correlation est significative à 0,001 & & & &
\end{tabular}

${ }^{* *}$, Correlation est significative à 0,001

Effet de la densité de plantation des espèces agroforiestières sur les rendements en biomasses et en graines: La densité de plantation des espèces agroforiestières n'a pas influencé les rendements en biomasse et en graines de haricots dans les 2 sites. La non différence entre ces deux traitements peut être lié au fait que les arbustes étaient moins âgés et pour cela aucune coupe était effectué pour restituer la matiere organique au sol dans les 2 sites. Les rendements en biomasse et graines issus de parcelles à faible densité
(FBD) et à haute densité (HTD) à Mushinga sur sol pauvre étaient suprieurs à ceux de parcelles temoins (TO0) (Figure 2). Une tendance contraire a été observée à Mulungu dans le sol relativement fertile. Cette difference observée entre ces traitements et le témoin serait due à l'apport de fertilisants dans ces traitements dans le site avec sol pauvre étant que les effets de la matière organique sont plus marqués dans le sol pauvre que fertile (Ntamwira et al., 2010). 


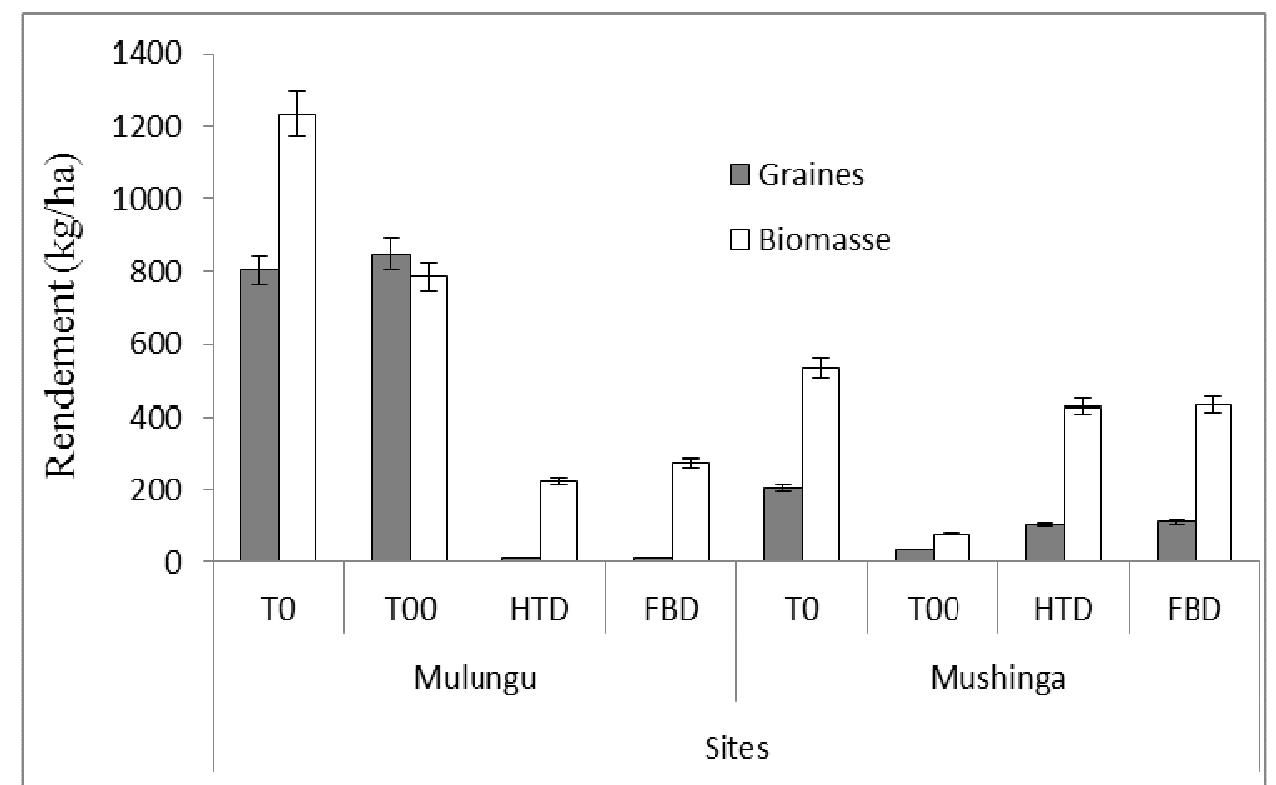

Figure. 2 : Effet de la densité de plantation des espèces agroforiestières sur le rendement en biomasse et en graines. HTD : haute densité des arbustes et FBD: Faible densité des arbustes.

Effet de la densité de plantation des espèces agroforiestières sur la teneur en fer et zinc dans les graines de harictos: Les résultats presentés par la figure 3 montrent que la densité de semis des arbustes agroforestiers n'a pas affecté la teneur en fer et zinc dans les graines de haricots. Elle était similaire dans les deux differentes densités de plantation et les témoins dans les 2 sites. Ce qui suggère que la teneur en micronitriments serait plus liée aux caractèrrs génétiques de chaque variété (Lestienne, 2004). En plus, les arbustes étaient encore trop petits, agés de 1 à 3 mois et ne pauvaient pas affecter la teneur en éléments dans le sol dans les différentes parcelles. L'effet de la densité de plantation des espèces agroforiestières sur la teneur en fer et zinc dans les graines de haricot est donné à la figure 3 .

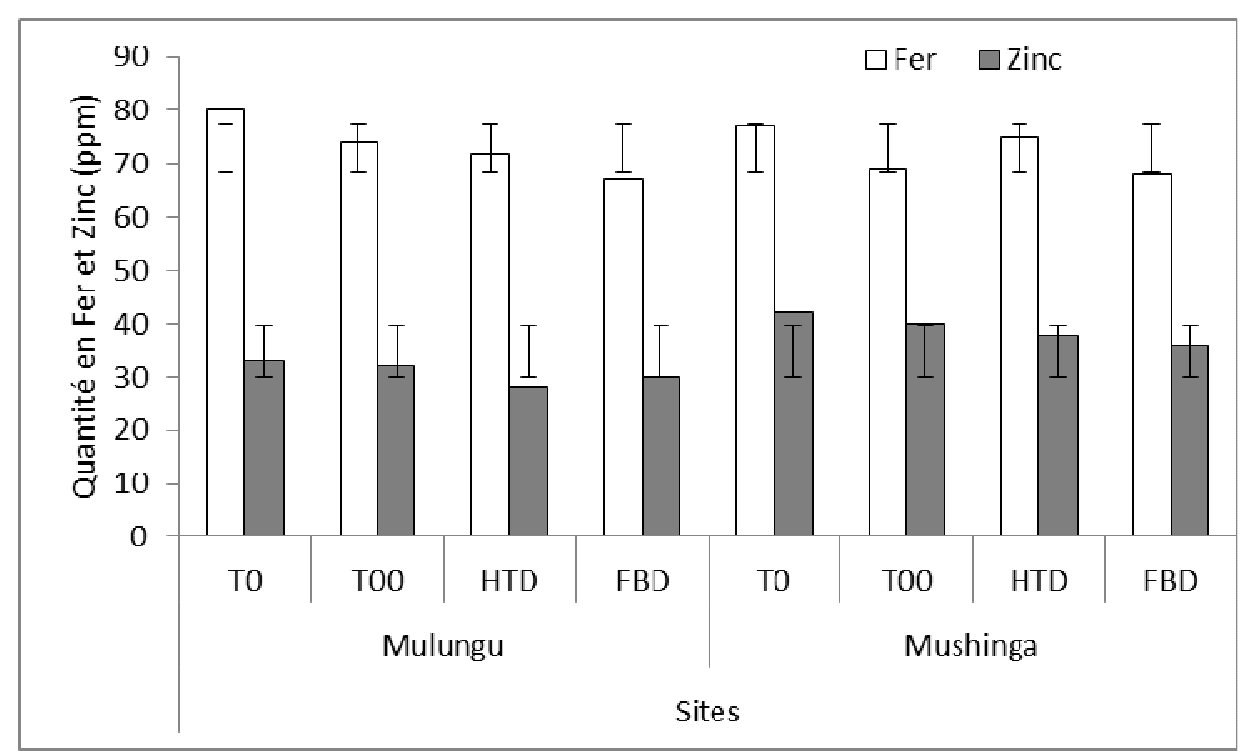

Figure 3 : Effet de la densité de plantation des espèces agroforiestièrres sur la teneur en fer et zinc dans les graines de haricots 
Effet de différentes combinaisons herbes-arbres sur le rendement moyen en biomasses et en graines de différentes variétés de haricots : Les résultats sur les rendements de quatre variétés de haricots utilisées pour cette expérimentation ont montré que le rendement en biomasse et en graines de haricots ont varié selon le type de sol et les variétés. La variété NAMULENGA a donné un rendement moyen en graines significativement plus élevé (140 kg.ha-1) alors que le rendement le plus bas (94 kg.ha-1) était produit par CUARANTINO dans le sol pauvre de Mushinga. Le classement des variétés selon leurs rendements dans ce site est le suivant: NAMULENGA $>$ M211 > CODMLB059 = CUARANTINO. Concernant le rendement moyen en biomasse, des variétés seraient classées dans l'ordre suivant: CODMLB059 $=$ M211 $=$ CUARANTINO $=$ NAMULENGA.

Aucune différence significative n'a été observée entre NAMULENGA, CUARANTINO 0817 et M211 dans le sol relativement fertile. Tandis que, CODMLB059 a donné des rendements en biomasse et en graines significativement inférieurs à ceux de ces 3 autres variétés. Le classement des variétés selon leurs rendements moyens en graines et biomasse dans ce site est le suivant : CUARANTINO = M211 = NAMULENGA > CODMLB059 (Fig. 4). Ce classement montre que toutes les variétés n'ont pas les mêmes potentiels de production, ce qui est similaire aux résultats menées en Ethiopie sur différentes variétés de haricots par Ashenafi \& Mekuria (2015). Ces résultats peuvent être expliqués par la variabilité qui existe au sein d'une même espèce quant à la capacité d'absorption d'éléments nutritifs du sol (Mulangwa, 2000. Néanmoins, il faut reconnaitre que les rendements en graines sont ici inferieurs aux rendements moyens généralement reportés pour ces variétés (Tableau 2) et cela serait dû aux conditions pluviométriques adverses ayant prévalu au cours de l'expérimentation et la faible fertilité de sites expérimentaux.

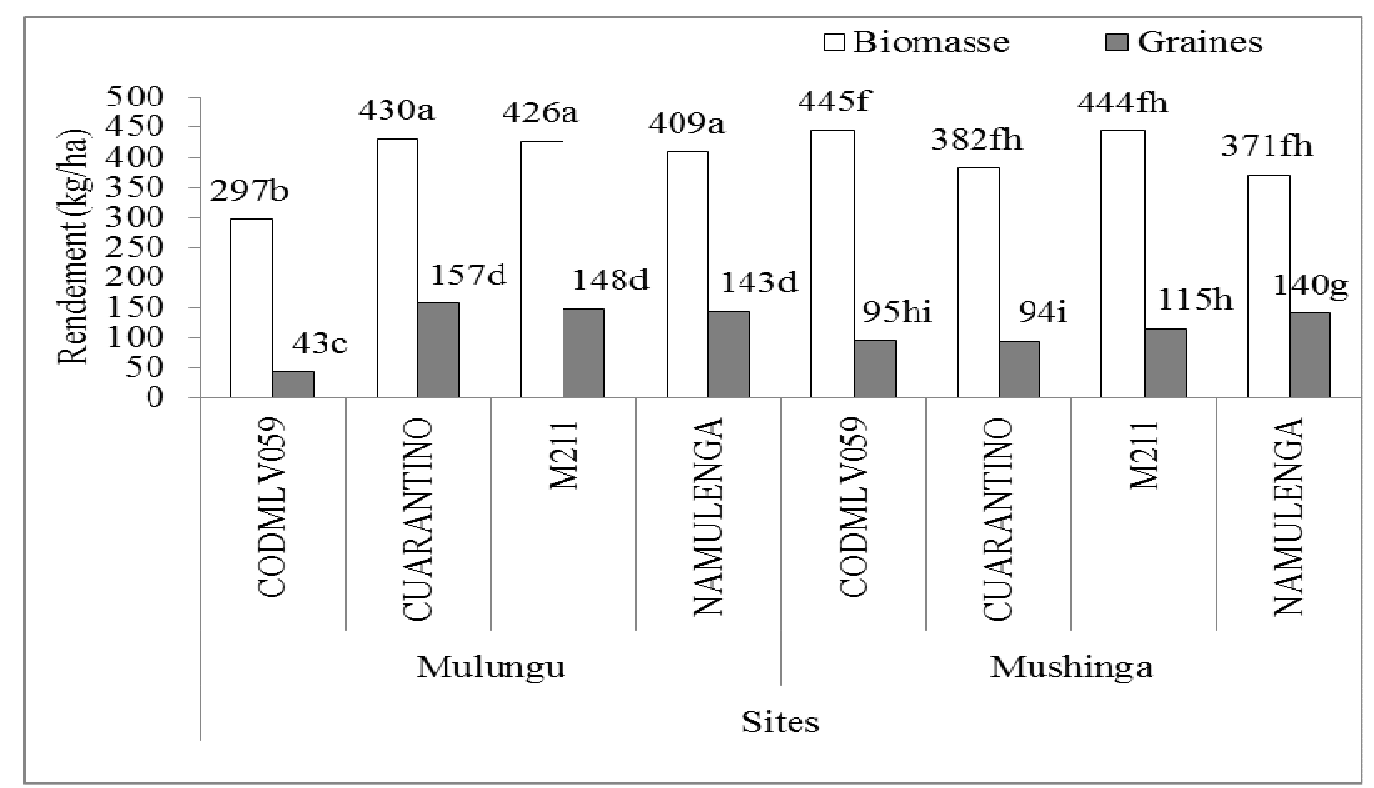

Figure 4. Rendement moyen en biomasse et en graines de 4 variétés de haricots dans les 2 sites. Par site, les barres avec les mêmes lettres ne sont pas significativement différentes selon le test de LSD (la plus petite différence significative), $p<0,05$.

\section{CONCLUSION}

Les différentes combinaisons herbes-arbres (système de culture en ATV) ont augmenté les rendements de haricots à Mushinga sur le sol pauvre. L'étude a montré que la microdose de fertilisants dans les conditions de cet essai a un grand effet sur l'amélioration du rendement en graines de haricots et que sans apport d'engrais le rendement était très bas dans le sol pauvre. Contrairement, la microdose d'engrais n'améliore pas le rendement, en biomasse et en graines de haricots dans le sol relativement fertile. Le système de culture en ATV n'a pas d'effet significatif sur les maladies de haricots. La teneur en fer dans les graines de haricots dans le sol pauvre de Mushinga était élevée dans les parcelles de culture en ATV que les parcelles témoins. La variété NAMULENGA serait mieux adaptée au sol de Mushinga par rapport aux trois autres. L'évaluation du carbone 
séquestré par les espèces agro forestières (graminées et arbustes) sera envisagée à moyen terme pour les graminées et à long terme pour les arbustes pour mètre

\section{REMERCIEMENTS}

L'auteur remercie le Centre International pour l'Agriculture Tropicale (CIAT) qui, à travers le Programme HarvestPlus, a financé les activités sur terrains. Les

\section{REFERENCES}

AFD (Agence française de développement), 2006. Le semis direct sur couverture végétale permanente (SCV), Paris, France.

Allen DJ, Ampofo JKO, Wortmann CS, 1996. Ravageurs, maladies et carences nutritives du haricot commun en Afrique. Guide pratique. Publication du CIAT, No. 265. 132 pp.

Ashenafi M. and Mekuria W, 2015. Effect of Faba Bean (Vicia faba L.) Varieties on Yield Attributes at Sinana and Agarfa Districts of Bale Zone, Southeastern Ethiopia. Jordan Journal of Biological Sciences, 8(4): 281 - 286.

Bouwmeester H, Van asten P, Ouma E, 2009. Mapping key variables of banana based cropping systems in the Great Lake Region, partial outcomes of the base-line and diagnostic surveys, International Institute of Tropical Agriculture, Ibadan, Nigeria. p50 pp.

CIALCA, 2010. CIALCA Baseline Survey Report, Consortium for Improving Agriculture-based Livelihoods in Central Africa. 129 pp.

CIALCA, 2009. Technical Progress Report November no 6, CIALCA II, January - December 2009. 85pp.

Civava MR, Malice M, Baudoin JP, 2012. Amélioration des agrosystèmes intégrant le haricot commun (Phaseolus vulgaris L.) au Sud-Kivu montagneux. Ed. Harmattan. Pp.69-92.

Dana GD, 1978. Development and Spread of highyielding varieties of wheat and rice in the Less Developed Nations.

DSRP, 200. La Monographie de la province du Sud-Kivu, Unité de Pilotage du Processus DSRP Kinshasa / Gombe. 122 pp.

FAO, 2012. Bonnes pratiques de Gestion Durable des Terres et des Eaux en Afrique du Nord, Projet grande muraille verte. $59 \mathrm{pp}$.

FAO, 2005. The importance of soil organic matter, FAO SOILS BULLETIN $80.95 \mathrm{pp}$.

FAO, 2003. Les engrais et leurs applications, Quatrième édition ISBN 92-5-104414-7. $77 \mathrm{pp}$. en évidence l'importance du système de culture en ATV sur la restauration de la fertilité de sol et la contribution à la lutte contre le réchauffement climatique

mêmes remerciements sont également adressés à I'INERA qui a mis à notre disposition des techniciens pour la collecte des données.

Fazle S, Rabbi MA, Peter V, Lockwood A, Heiko DA, 2010. How do microaggregates stabilize soil organic matter? World Congress of Soil Science, Soil Solutions for a Changing World. Pp 109111.

GenStat, 2010. GenStat Discovery, Edition 4. Available at: http://www.vsni.co.uk/software/free-touse/discovery/genstat-discovery.

Husson O, Charpentier H, Razanamparany C, Moussa N, Michellon R, Naudin K, Razafintsalama H, Rakotoarinivo C, Rakotondramanana, Seguy S, 2008. Fiches techniques de plantes de couverture : Graminées pérennes, Manuel pratique du semis direct à Madagascar, Volume III, chapitre 4. 20 pp.

Imrani N, ouazzani AC, Chliyeh M, Touati J, Ouazzani TA, Benkirane Ret Douira A, 2014. Effet de la fertilisation par différents niveaux de N P K sur le développement des maladies foliaires du riz. Journal of Animal and Plant Sciences 23 (1): 3601-3625.

INERA, 2006, Caractérisation de haricot commun. (Phaseolus vulgaris L.). Programme national Haricot (PNH), centre de Mulungu. Année 2006 A et B. 58 pp.

Jiao Y, Whalen JK, Hendershot WH, 2006. No-tillage and manure applications increase aggregation and improve nutrient retention in a sandy-loam soil, Geoderma 92:111-123.

Kaho $F$, Yemefack M, Feujio-Teguefouet $P$, Tchantchaouang JC, 2011. Effet combiné des feuilles de Tithonia diversifolia et des engrais inorganiques sur les rendements du maïs et les propriétés d'un sol ferralitique au Centre Cameroun. Tropicultura 29 (1): 39-45.

Kanyenga AL, Kasongo EL, M, Kizungu RV, Nachigera GM et Kalonji KM, 2016. Effect of climate change on common bean (Phaseolus vulgaris) crop production: determination of the optimum planting period in midlands and highlands zones of the Democratic Republic of Congo. Global, 
Journal of Agricutural, Research and Reviews 4 (1): 390-399.

Lestienne I, 2004. Contribution à l'étude de la biodisponibilité du fer et du zinc dans le grain de mil et conditions d'amélioration dans les aliments de complément. Thèse de Docteur, Université Montpellier II, France. 245 pp.

Lunze L, Mathew M, Abang, Buruchara R, Michael A, Ugen, Nabahungu NL, Gideon O, Rachier, Ngongo M, Idupulapati R, 2012. Integrated Soil Fertility Management in Bean- Based Cropping Systems of Eastern, Central and Southern Africa, Soil Fertility Improvement and Integrated Nutrient Management - A Global Perspective, Dr, Joann Whalen (Ed,),. $35 \mathrm{pp}$.

Lunze L, 2000. Possibilités de gestion de la fertilité des sols au Sud-Kivu montagneux. Cahier du Centre d'études et de recherche pour la promotion rurale et la paix (CERPRU) 14 : 28-34.

Masi, 1982. Classification des sols du Zaïre dans la province du Kivu, Rapport final de classification des sols, projet de soutien de I'INERA, 660.0064 .

Mulangwa N, 2001. L'efficacité de Tithonia diversifolia en tant qu'amendement édaphique pour la culture du haricot commun (Phaseolus vulgaris L.) in PABRA Millenium Workshop Novotel Mount Meru, Arusha, Tanzania 28 May - 1 June 2001. Pp 264-276.

Mulangwa N, 2000. Type de croissance et réponse du haricot (Phaseolus vulgaris L.) à la fumure. Cahier du Centre d'études et de recherche pour la promotion rurale et la paix (CERPRU) 14 : 4346.

Muriel F, 2007. Gestion des matières organiques dans les sols cultivés en Région wallonne : avantages agronomiques, avantages environnementaux et séquestration du carbone, Mémoire inédit, Université Libre de Bruxelles. 102 pp.

Ngongo M. and Lunze L, 2000. Espèce d'herbe dominante comme indice de la productivité du sol et de la réponse du haricot commun à l'application du compost. African Crop Science Journal 8 (3): 251-261.

Ntamwira J, Dowiya ZB, Katunga M, Van Asten PJA. and Blomme G, 2010. The effect of application of organic matter during planting on growth of an East African Highland banana on two contrasting soils in South-Kivu, Eastern DR Congo. Tree and Forestry Science and Biotechnology 4 (special issue 2) Global Science Book.15-16 p.
Ntamwira J, Pypers $P$, van Asten $P$, Vanlauwe B, Ruhigwa B, Lepoint $P$, Dhed'a B, Monde T, Blomme G, 2014. Effect of banana leaf pruning on banana and legume yield under intercropping in farmers' fields in eastern Democratic Republic of Congo, Journal of Horticulture and Forestry 6(9): $72-80$.

Pyame D, 2015. Propriétés agronomiques et potentiel d'atténuation des changements climatiques d'une agro-forêt de type "culture en assiettes sous tapis vert», en restauration de sols dégradés, à Kisangani (RD Congo), Thèse de Docteur en Sciences Agronomiques, Université de Kisangani, RD Congo. 192 pp.

Pypers $P$, Sanginga J, Kasereka B, Walangululu M, Vanlauwe B, 2010. Increased productivity through integrated soil fertility management in cassava-legume intercropping systems in the highlands of Sud-Kivu, DR Congo. Field Crops Research 120 (1): 76-85.

Razafindramanan NC, Douzet JM, Barthès $B$, Rabeharisoa L, Albrecht A, 2012. Evaluation des effets de systèmes de semis direct à couverture végétale pérenne (SCV) sur l'érosion hydrique et la production agricole sur les Hautes-Terres de Antsirabe (Madagascar). $12 \mathrm{pp}$.

Razafimbelo T, Albrecht A, Basile I, Borschneck D, Bourgeon G, Feller C, Ferrer H, Michellon R, Moussa N, Muller B, Oliver R, Razanamparany C, Seguy L, Swarc M, 2006. Effet de différents systèmes de culture à couverture végétale sur le stockage du carbone dans un sol argileux des Hautes Terres de Madagascar. Etude et Gestion des sols 13: 113-127.

Reij C. and Thiombiano T, 2003. Développement rural et environnement au Burkina Faso: la réhabilitation de la capacité productive des terroirs sur la partie nord du Plateau Central entre 1980 et 2001, Fee University of Amsterdam, The Netherlands . 80 pp.

Sallah PYK, Mukakalisa S, Nyombayire A, Mutanyagwa $P, 2009$. Response of two maize varieties to density and nitrogen fertilizer in the highland zone of Rwanda. Journal of Applied Biosciences 20: 1194-1202. Sana DF, 2011. Diversités phénotypiques et moléculaire des microsymbiotes du Sulla du nord (Hédysarum Coronarium L.) et sélection de souches rhizobiales efficientes. Thèse de Docteur en Sciences Agronomiques, Institut national agronomique de Tunisie. 145 pp. 
Sanginga N. and Woomer PL, 2009. Integrated soil fertility management in Africa: principles, practices and developmental process, $270 \mathrm{pp}$.

Six J, Conant T, Paul A, Paustian K, 2002. Stabilization mechanisms of soil organic matter: Implications for C-saturation of soils. Plant and Soil 241: 155176.

Stangoulis J, 2010. Technical aspects of zinc and iron analysis in biofortification of the staple food crops, wheat and rice. World Congress of Soil
Science, Soil Solutions for a Changing World, Brisbane, Australia. Vol. 42. Pp 1-6.

Van Schoonhoven A. and Pastor-Corrales MA, 1992. Système standard pour l'évaluation du germoplasme du haricot, CIAT (Centro International de Agricultura Tropical). Publication du CIAT N²07. 49 pp.

Wouters JFR, Wakana M, Opdecamp L, 1986. Sensibilité du haricot Phaseolus vulgaris à la concentration en aluminium de sols de la région des Grands Lacs. Tropicultura 4 (1) : 20-26. 